\title{
A Comprehensive Review on Phytochemistry and Pharmacological Activities of Clinacanthus nutans (Burm.f.) Lindau
}

\author{
Leng Wei Khoo, ${ }^{1}$ Siew Audrey Kow, ${ }^{2}$ Ming Tatt Lee $\mathbb{D}^{\circ},{ }^{3}$ Chin Ping Tan $\left(\mathbb{D},{ }^{4}\right.$ \\ Khozirah Shaari, ${ }^{5}$ Chau Ling Tham, ${ }^{2}$ and Faridah Abas $\mathbb{C}^{1,5}$ \\ ${ }^{1}$ Department of Food Science, Faculty of Food Science and Technology, Universiti Putra Malaysia, 43400 Serdang, Selangor, Malaysia \\ ${ }^{2}$ Department Biomedical Science, Faculty of Medicine and Health Sciences, Universiti Putra Malaysia, 43400 Serdang, \\ Selangor, Malaysia \\ ${ }^{3}$ Faculty of Pharmaceutical Sciences, UCSI University Kuala Lumpur Campus, Jalan Menara Gading, UCSI Heights (Taman \\ Connaught), Cheras, 56000 Kuala Lumpur, Malaysia \\ ${ }^{4}$ Department of Food Technology, Faculty of Food Science and Technology, Universiti Putra Malaysia, 43400 Serdang, \\ Selangor, Malaysia \\ ${ }^{5}$ Laboratory of Natural Products, Institute of Bioscience, Universiti Putra Malaysia, 43400 Serdang, Selangor, Malaysia
}

Correspondence should be addressed to Faridah Abas; faridah_abas@upm.edu.my

Received 30 January 2018; Accepted 3 April 2018; Published 4 July 2018

Academic Editor: Hajime Nakae

Copyright (C) 2018 Leng Wei Khoo et al. This is an open access article distributed under the Creative Commons Attribution License, which permits unrestricted use, distribution, and reproduction in any medium, provided the original work is properly cited.

\begin{abstract}
Clinacanthus nutans (Burm.f.) Lindau (Acanthaceae), commonly known as Sabah snake grass, is a vegetable and a well-known herb that is considered an alternative medicine for insect bites, skin rashes, herpes infection, inflammation, and cancer and for health benefits. Current review aims to provide a well-tabulated repository of the phytochemical screening, identification and quantification, and the pharmacological information of $C$. nutans according to the experimental design and the plant preparation methods which make it outstanding compared to existing reviews. This review has documented valuable data obtained from all accessible library databases and electronic searches. For the first time we analyzed the presence of flavonoids, triterpenoids, steroids, phytosterols, and glycosides in C. nutans based on the results from phytochemical screening which are then further confirmed by conventional phytochemical isolation methods and advanced spectroscopic techniques. Phytochemical quantification further illustrated that $C$. nutans is a good source of phenolics and flavonoids. Pharmacological studies on C. nutans revealed that its polar extract could be a promising anti-inflammation, antiviral, anticancer, immune and neuromodulating, and plasmid DNA protective agent; that its semipolar extract could be a promising antiviral, anticancer, and wound healing agent; and that its nonpolar extract could be an excellent anticancer agent.
\end{abstract}

\section{Introduction}

Utilizing plants as a source of medicines has been practiced for a long time, especially in developing countries where drugs are usually inaccessible or costly, obligating people to use traditional remedies. Vast development in investigating the herbal plants as alternative therapeutic agents for different type of diseases is notable, starting from the late 1980s and continuing in recent years. Malaysia, a country with rich biodiversity, has been gifted with valuable medicinal plants resources in its tropical rainforest. Among them,
Acanthaceae is considered as one of the leading families of the dicotyledonous flowering plants and consist of 250 genera and approximately 2500 species [1]. This family is mainly distributed in Indonesia, Malaysia, Africa, Brazil, and Central America and possesses numerous high medicinal value species $[2,3]$.

Clinacanthus nutans (Burm.f.) Lindau, which belongs to the Acanthaceae family, is a very well-known traditional herb and vegetable in Southeast Asia countries and has been chosen for this review study [4]. It has a few vernacular names 
such as "belalai gajah" or "pokok stawa ular" (Malay), "e zui hua" or "you dun cao" (Chinese), "payayor" or "slaed pang pon" (Thai), and "daun dandang gendis" or "kitajam" (Javanese). Since a long time ago, this plant is famous as an antidote for snake bites, herpes infection, skin infection, cancer, burns and scalds, dysentery, and diabetes in Thailand, Indonesia, and Malaysia [5-8]. Numerous studies on this plant have been published by researchers from Thailand since 1967. Since 1987, C. nutans has become one of the herbs recommended for use in hospitals and was included in the Primary Health Care Programme by the Government of Thailand $[9,10]$. The most used part of the plant was the leaf, and the common prescription method was decocting the plant with water for oral ingestion or soaking it in the alcohol for a week for topical application to an affected area [11]. In 2011, this plant began gaining popularity in Malaysia, and various types of $C$. nutans related studies have been conducted by Malaysian scientists.

Authors noticed the publication of reviews on this plant, where some of them also discussed the topics chosen in this paper $[2,12-16]$. However, authors target to provide a well-managed summary and possible recommendation on the existing studies so that the readers could acquire much information regarding the current and future direction of this plant from present review just by a glance. Thus, a well-organized and critical review paper on this plant is in demand to reduce redundant studies and allow new insight into the research direction to be made. Therefore, instead of discussing a few selected studies, present review discusses and covers thoroughly the previous C. nutans studies related to the topics chosen. The details such as preand postharvesting preparation methods of the plant extract as well as the experiment design applied in the studies of phytochemical screening, identification and quantification, and pharmacological activities of $C$. nutans are systematically categorized, compared, and summarized. Information on the preharvesting (plant age and plant origin) and postharvesting (plant part used, drying method, extraction method, extraction solvent, and storage duration) methods of the plant as well as on the experiment design employed was considered and was emphasized in the discussion section which makes present review different from the others. Different plant preparation methods and experimental designs will result in the generation of different conclusions, even for the same plant. Thus, including and correlating all variable factors and parameters in the discussion will help to determine the pro and cons of each study and lead to a better summary in the end. We hypothesized that, through all these efforts, a good summary, precautionary step, challenge, limitation, new idea, or a clearer future perspective may be initiated.

\section{Phytochemistry}

2.1. Phytochemical Screening. For the last few decades, the study of plants has progressed rapidly [17]. Plants contain both primary and secondary metabolites. Primary metabolites are those chemical constituents that are inherently present in most organisms and have a direct involvement in plant growth [18]. On the other hand, secondary metabolites are the bioactive phytochemicals that are normally produced by a plant in response to specific environmental stresses and that are considered to possess additional health benefits [18]. Throughout the years, the common practice of researchers was started with the screening of plant. Phytochemical screening tests, thin layer chromatography (TLC) and Fourier transform infrared spectroscopy (FTIR) are among the most popular methods used as they are easy to conduct, of low cost, and time-effective. These methods are able to provide guidelines on the class and functional groups of the chemical constituents that are present in the plant.

Table 1 lists the occurrence of chemical classes in C. nutans extract, as determined through specific phytochemical screening tests. The phytochemical screening tests adopted include Mayer's reagent, modified Dragendorff's reagent, or the Wagner test for alkaloid detection; the frothing test for saponin, the alkaline reagent, Shinoda, or lead acetate tests for flavonoids; the frothing, ferric chloride, potassium dichromate, or gelatin tests for tannins; the copper acetate test for diterpenes; Salkowski's test, or Liebermann-Burchard test for phytosterols; the iron (III) chloride test for phenolic compounds; the pseudoindicans test for iridoids; Grignard reagent, Baljet, Keller-Kiliani, or Molisch tests for glycosides; Molisch or Fehling tests for carbohydrates; Salkowski's test for steroids and sterols; and the biuret test for proteins and amino acids $[6,19-22]$. As a summary, flavonoids, triterpenoids, steroids, phytosterols, and glycosides are the phytochemical classes that are most likely present in C. nutans extract when the plant is extracted by a polar solvent, such as water, methanol, ethanol, or an aqueous organic solvents or by a semipolar (e.g., chloroform) solvent. The presence of alkaloids, saponins, and tannins in C. nutans depends on the plant origin and the postharvesting method used. For instance, alkaloids were typically present in water and chloroform leaf extracts and were absent in methanol and aqueous methanol leaf extracts collected from Malaysia. By contrast, saponins were present in methanol extract cultivated in Malaysia and absent in water extract from Indonesia and chloroform and aqueous methanol extracts from Malaysia. Tannins were found in leaves extracted with water, aqueous methanol, and chloroform, but not those extracted with methanol. For the presence of quinones, iridoids, carbohydrates and protein, and amino acid, further confirmation is required as there has been only one study conducted on each of them to date.

In 2012, Roeslan and his teams [11] compared leaves from two different sources, Thailand and Indonesia, using TLC separation method. Their findings showed that there was a quality difference and indicated that the quality of the sample from Indonesia was better, as this sample was thicker than the sample from Thailand. Chelyn et al. [23] used the HPTLC method to screen for the presence of C-glycosyl flavones in three $C$. nutans plant samples that underwent the same postharvesting processing but that were from three different geographical locations in Malaysia: Taiping (Perak), Kota Tinggi (Johor), and Sendayan (Negeri Sembilan). All the results indicated that schaftoside was present in $C$. nutans collected from all the investigated locations, whereas isoorientin, isovitexin, orientin, and vitexin were found only in the plants harvested from Perak and Johor, not in the 
TABLE 1: Phytochemical screening test of C. nutans.

\begin{tabular}{|c|c|c|c|}
\hline Chemical class & Origin & Extract & Present/Absent \\
\hline \multirow{4}{*}{ Alkaloid } & Indonesia $^{1}$ & Leaf-water extract $^{1}$ & Present $^{1}$ \\
\hline & Malaysia $^{2,3,5}$ & Leaf-100\% methanol extract ${ }^{2,5}$ & Absent $^{2,5}$ \\
\hline & & Leaf- $100 \%$ chloroform extract ${ }^{3}$ & Presence $^{3}$ \\
\hline & & Leaf-70\% methanol extract ${ }^{4}$ & Absent $^{4}$ \\
\hline \multirow{4}{*}{ Saponin } & Indonesia $^{1}$ & Leaf-water extract ${ }^{1}$ & Absent $^{1}$ \\
\hline & Malaysia $^{2,3,4,5}$ & Leaf- $100 \%$ methanol extract ${ }^{2,5}$ & Present ${ }^{2,5}$ \\
\hline & & Leaf- $100 \%$ chloroform extract ${ }^{3}$ & Absent $^{3}$ \\
\hline & & Leaf-70\% methanol extract ${ }^{4}$ & Absent $^{4}$ \\
\hline \multirow{4}{*}{ Flavonoids } & Indonesia $^{1}$ & Leaf-water extract ${ }^{1}$ & Present $^{1}$ \\
\hline & Malaysia $^{2,3,4,5}$ & Leaf-100\% methanol extract ${ }^{2,5}$ & Present ${ }^{2,5}$ \\
\hline & & Leaf- $100 \%$ chloroform extract ${ }^{3}$ & Present $^{4}$ \\
\hline & & Leaf-70\% methanol extract ${ }^{4}$ & Present $^{3}$ \\
\hline \multirow{2}{*}{ Triterpenoids } & Indonesia $^{1}$ & Leaf-water extract $^{1}$ & Present $^{1}$ \\
\hline & Malaysia $^{5}$ & Leaf- $100 \%$ methanol extract ${ }^{5}$ & Present $^{2}$ \\
\hline Diterpenes & Malaysia $^{2}$ & Leaf- $100 \%$ methanol extract ${ }^{2}$ & Present \\
\hline \multirow{4}{*}{ Steroids } & Indonesia $^{1}$ & Leaf-water extract ${ }^{1}$ & Present $^{1}$ \\
\hline & Malaysia $^{4,5}$ & Leaf- $70 \%$ methanol extract ${ }^{4}$ & Present ${ }^{2,3}$ \\
\hline & Thailand $^{7}$ & Leaf- $100 \%$ methanol extract ${ }^{5}$ & Present $^{5}$ \\
\hline & & Stem-Light petroleum extract ${ }^{7}$ & Present $^{7}$ \\
\hline \multirow{2}{*}{ Phytosterol } & Malaysia $^{2,4}$ & Leaf-100\% methanol extract ${ }^{2}$ & Present $^{1}$ \\
\hline & & Leaf- $70 \%$ methanol extract $^{4}$ & Present $^{2}$ \\
\hline \multirow{4}{*}{ Tannin } & Indonesia $^{1}$ & Leaf-water extract ${ }^{1}$ & Present $^{1}$ \\
\hline & Malaysia $2,3,4,5$ & Leaf-100\% methanol extract ${ }^{2,5}$ & Absent $^{2,5}$ \\
\hline & & Leaf- $100 \%$ chloroform extract ${ }^{3}$ & Present $^{3}$ \\
\hline & & Leaf- $70 \%$ methanol extract ${ }^{4}$ & Present ${ }^{4}$ \\
\hline Quinone & Indonesia $^{1}$ & Leaf-water extract ${ }^{1}$ & Absent \\
\hline Phenolic compound & Malaysia $^{2}$ & Leaf- $100 \%$ methanol extract ${ }^{2}$ & Present \\
\hline \multirow{2}{*}{ Glycosides } & Malaysia $^{3,4}$ & Leaf- $100 \%$ chloroform extract ${ }^{3}$ & Present $^{1}$ \\
\hline & & Leaf-70\% methanol extract ${ }^{4}$ & Present $^{2}$ \\
\hline Iridoids & Thailand $^{6}$ & Leaf $^{6}$ & Present $^{6}$ \\
\hline Carbohydrates & Malaysia $^{4}$ & Leaf- $70 \%$ methanol extract $^{4}$ & Present $^{4}$ \\
\hline Protein and amino acids & Malaysia $^{4}$ & Leaf- $70 \%$ methanol extract ${ }^{4}$ & Present ${ }^{4}$ \\
\hline
\end{tabular}

Reference. ${ }^{1}$ Nurulita et al. [25]; ${ }^{2}$ Yang et al. [6]; ${ }^{3}$ Goonasakaran [26]; ${ }^{4}$ Sekar and Rashid [22]; ${ }^{5}$ Abdul Rahim et al. [19]; ${ }^{6}$ Keawpradub and Purintrapiban [20]; ${ }^{7}$ Dampawan et al. [27].

sample harvested from Negeri Sembilan. Tiew et al. [24] used UV-visible and FTIR spectrophotometry for the functional group identification of $C$. nutans leaves dried under the shade and macerated with methanol. Based on the results obtained, the extract showed two major bands $240-400 \mathrm{~nm}$ and the presence of an $-\mathrm{OH}$ band and a $\mathrm{C}=\mathrm{O}$ stretch suggests that the constituents in the extract have $-\mathrm{OH}$ group and $\mathrm{C}=\mathrm{O}$ functional groups.

2.2. Phytochemical Identification. Phytochemical screening methods can only provide a clue of the chemical class present in the plant. To further validate the identity of the compounds, column chromatography (isolation) and TLC (separation and purity) together with nuclear magnetic resonance (NMR) spectroscopy (identification and structural elucidation) are the most conventionally used techniques. In addition, the emergence of advanced analytical tools such as liquid chromatography mass spectrometry (LCMS), gas chromatography mass spectrometry (GCMS), and NMR spectroscopy provides researchers with useful alternatives for identification.

\subsubsection{Phytochemical Identification Using Column Chromatog-} raphy-Spectroscopy Analysis. A summary of the phytochemicals that have been identified in this plant is provided in Tables 2(a) and 2(b). Table 2(a) displays those compounds that have been isolated through column chromatography and identified using spectroscopic analysis, the results from which were tabulated according to the phytochemical class. To date, the pure compounds that have been isolated from C. nutans include 2 triterpenoids; 6 phytosterols; 8 phenolics, including $7 \mathrm{C}$-glycosyl flavones and 1 phenolic acid; 5 sulfurcontaining glucosides; 8 sulfur-containing compounds; 7 lipid related compounds; and 8 chlorophyll derivatives. 


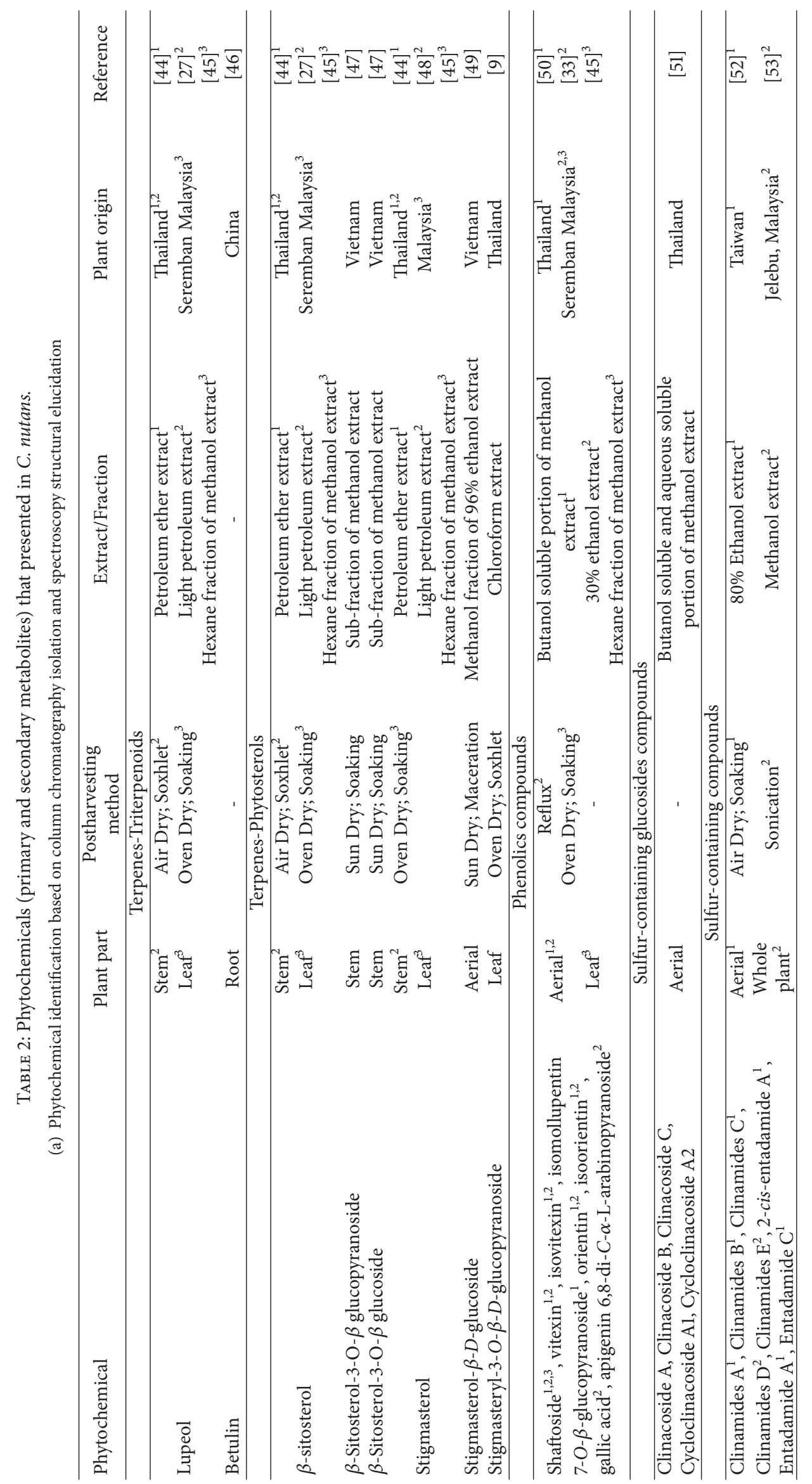




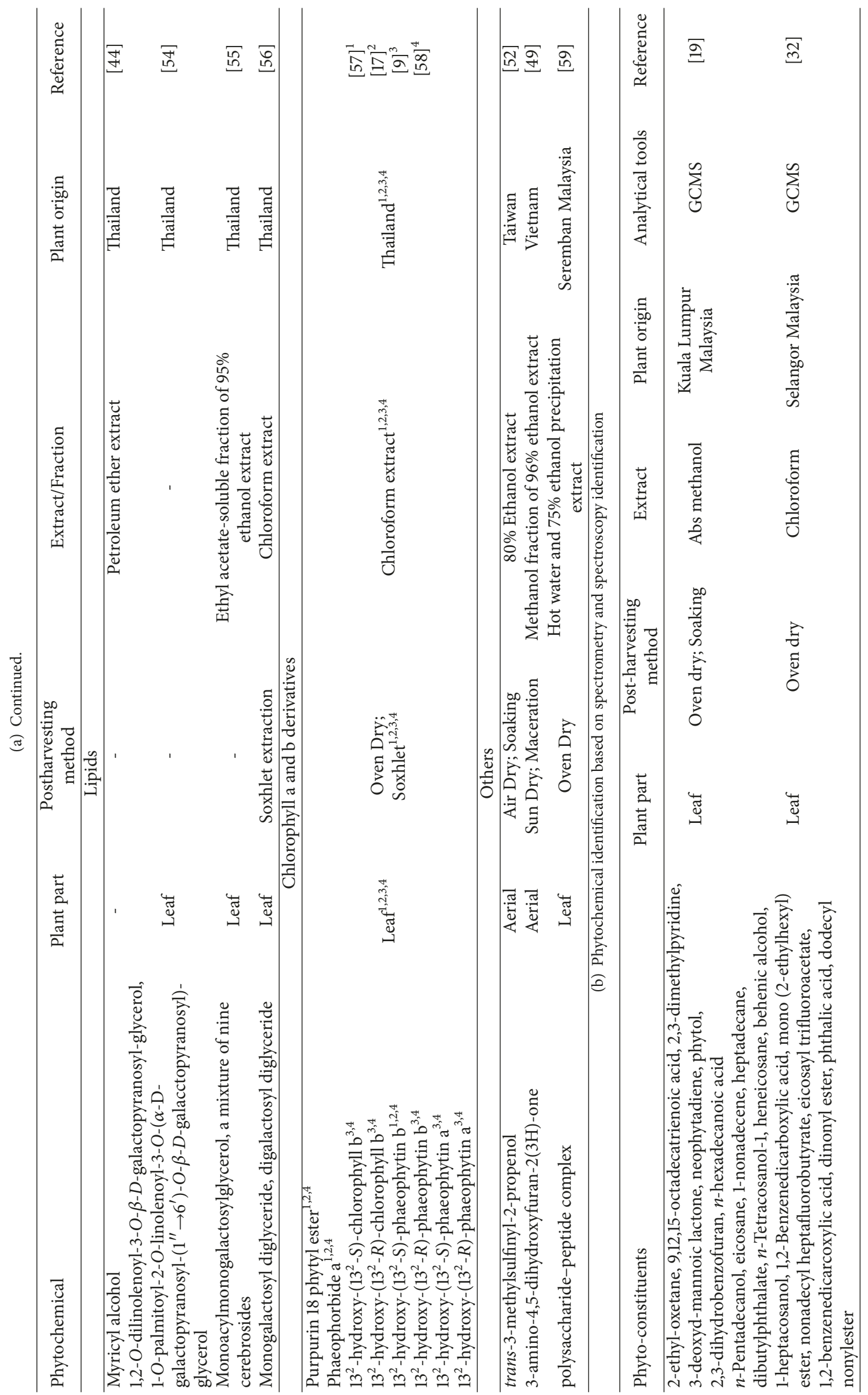




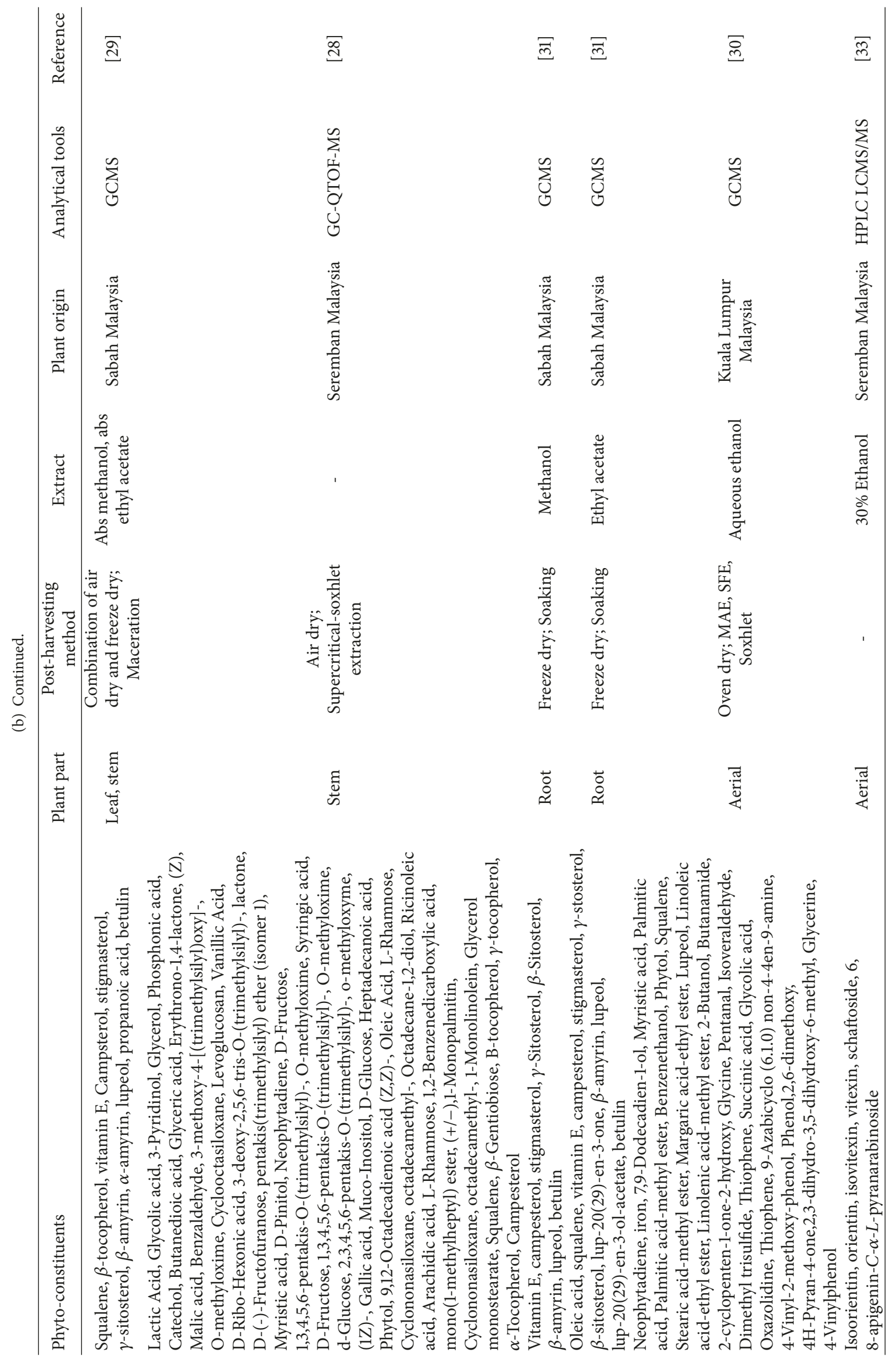




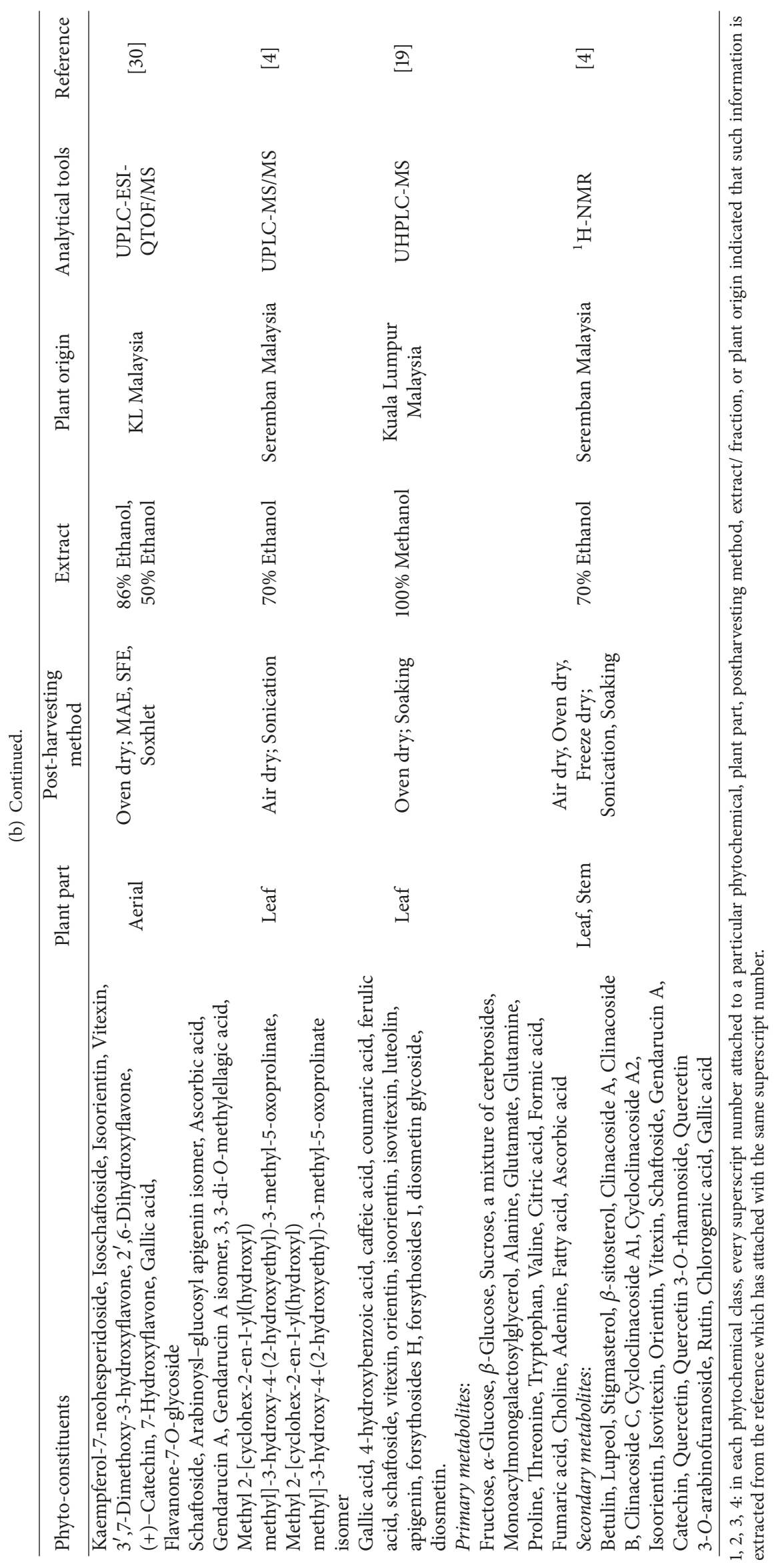


2.2.2. Phytochemical Identification Using Advanced Spectrometric and Spectroscopic Analysis. Meanwhile, Table 2(b) shows a list of compounds identified according to the type of the advanced spectrometric and spectroscopic analytical tools used. There are a total of 6 research teams that have used GCMS for compound identification [19, 28-32], 4 research teams that have used LCMS analytical tool [4, 19, 30, 33], and 1 research team that has used proton and $J$-resolved NMR approaches [4]. Based on GCMS identification results, Cheong et al. [29] found a rich variety of triterpenoids and phytosterols in the extract. Yong et al. [32] showed that of the 14 phytochemicals identified in the chloroform extract, 1,2benzenedicarboxylic acid, mono(2-ethylhexyl)ester, a common plasticizer, was the most abundant compound. Mustapa et al. [30] found that phytol was the main compound in the microwave (MAE) and Soxhlet assisted extracts, while palmitic acid was dominant in the supercritical fluid assisted (SFE) extract. A study by Abdul Rahim et al. [19] identified a total of 39 peaks, but only 8 major compounds have been listed in Table 2(b). In addition, Alam et al. [28] have successfully identified a total of 48 compounds, the most abundant of which were neophytadiene, glycolic acid, phosphonic acid, catechol, butanedioic acid, vanillic acid, gallic acid, pinitol, phytol, squalene, tocopherols, and campesterol. Moreover, Teoh et al. [31] have found that the ethyl acetate root extract possessed more compounds than the methanol root extract. Among the compounds identified, lupeol was the main compound present in both extracts.

Using LCMS in negative ionization mode, Huang et al. [33] successfully identified 6 C-glycosyl flavones, the research team of Khoo et al. [4] was able to identify 8 compounds, Mustapa et al. [30] team have found 10 phenolic compounds from the aqueous ethanolic extract, and Abdul Rahim et al. [19] have detected 16 phenolic compounds. Khoo et al. [4] have also tentatively identified 20 primary metabolites and 22 secondary metabolites in $70 \%$ ethanol extract through proton and $J$-resolved NMR spectroscopy.

\subsection{Phytochemical and Nutrient Content. Environmental} condition, geographical location, cultivation practices, genetics, pre- and postharvesting methods practiced and some other unexpected factors will cause phytochemical level variations in a plant even in the same species [18]. Thus, this section reveals the phytochemical content of $C$. nutans including the total phenolic content (TPC) in Table 3, the total flavonoid content (TFC) in Table 4, and the nutritional composition of C. nutans in Table 5. Generally, the phytochemical content of C. nutans has been evaluated using simple chemical assay or high performance liquid chromatography (HPLC) methods. Compared to other tests, determination of TPC and TFC is preferred due to the interest in the therapeutic benefit imparted by phenolics and flavonoid; in addition, they are easy, time-effective, and cost-effective and well-established methods.

The Folin-Ciocalteu method is the most established method for determining the total phenolic content of $C$. nutans and gallic acid or, in some cases, tannic acids are the most common standards used for TPC calibration. Values are normally expressed in mg gallic acid equivalent (GAE)/g dry weight (DW) or dry material (DM). Total phenolic content is based on a colorimetric reaction between easily oxidized polyphenols or hydroxylated aromatic compounds and phosphotungsten-polymolybdic acid [34]. The redox reaction results in the formation of blue chromophores consisting of phosphotungstic-phosphomolybdenum complexes, which have a maximum absorption at $765 \mathrm{~nm}$ and are proportional to the total quantity of phenolic compounds [34]. Among the TPC tests conducted, the chloroform fraction prepared by Hamid and Yahaya [35] from a Malaysian farm that underwent sonication was detected to have the highest TPC, with $119.29 \pm 0.07 \mathrm{mg}$ GAE/g DW. In addition, Lusia Barek et al. [36] also illustrated that an unfermented leaf subjected to drying in a microwave oven and infusion in water for $20 \mathrm{~min}$ retained a high TPC value of $177.80 \pm$ $19.10 \mathrm{mg}$ tannic acid equivalent (TAE)/L.

The flavonoid level in $C$. nutans was mostly determined by the aluminum chloride $\left(\mathrm{AlCl}_{3}\right)$ method. Essentially, the detection of flavonoid (the colored complexes) is conducted using the reaction between $\mathrm{AlCl}_{3}$ and the carbonyl and hydroxyl groups of flavonoids in an alkaline solution [18]. As shown by the results in Table 4, TFC was determined using quercetin as the positive control and the results were expressed in $\mathrm{mg}$ quercetin equivalent $(\mathrm{QE}) / \mathrm{g}$ dry weight (DW) sample, although there were also some studies that used catechin, rutin, or butylated hydroxytoluene (BHT) as the positive control. Again, the chloroform fraction of $C$. nutans that underwent sonicated extraction was able to retain more TFC (937.67 $\pm 0.02 \mathrm{mg} \mathrm{BHTE} / \mathrm{g})$ than those undergoing other combination of pre- and postharvesting methods [35].

Other phytochemical content studies on C. nutans included hydroxycinnamic acid content determination using Arnow's reagent. The results suggested that oven-dried and hot water extracted $C$. nutans extract possessed $0.91 \pm 0.01 \mathrm{mg}$ caffeic acid equivalent/g dry material (mg CAE/g DW) [37]. For the chlorophyll content of C. nutans, Raya et al. [38] found that, compared to mature stem $(32.27 \mathrm{mg} / 100 \mathrm{~g})$ or plant sample that kept for 4 days (17.97 $\mathrm{mg} / 100 \mathrm{~g})$, a young leaf or plant sample subjected to 1 day of storage possessed a higher chlorophyll content with $64.35 \mathrm{mg} / 100 \mathrm{~g}$ and $78.83 \mathrm{mg} / 100 \mathrm{~g}$, respectively. By contrast, Mustapa et al. [30] found that the absolute ethanol aerial extract of $C$. nutans possessed a higher chlorophyll content $(1.30 \mathrm{~g} / \mathrm{g} \mathrm{DM})$ than absolute acetone aerial extract $(1.06 \mathrm{~g} / \mathrm{g}$ DM). On the other hand, Raya et al. [38] showed that C. nutans leaves harvested at a young stage had a higher content of ascorbic acid $(0.38250 \mathrm{mg} / 100 \mathrm{~g})$. The results also revealed that increasing the storage duration from 1 day to 4 days caused a reduction in the ascorbic content of the extract from $0.49833 \mathrm{mg} / 100 \mathrm{~g}$ to $0.23083 \mathrm{mg} / 100 \mathrm{~g}$. For phytosterol determination using Liebermann-Burchard reagent, one study showed that increasing the organic solvent ratio in the solvent mixture will result in a decrease in the phytosterol content [30]. This study also suggested that the supercritical fluid extraction (SFE) method allowed higher phytosterol retention $(1.35 \pm 0.12 \mathrm{mg}$ of $\beta$-sitosterol (BS)/g $\mathrm{DM})$, followed by pressurized-microwave assisted extraction (p-MAE) for the $86 \%$ ethanolic extract $(1.19 \pm 0.22 \mathrm{mg} \mathrm{BS} / \mathrm{g}$ $\mathrm{DM}$ ), microwave assisted extraction (MAE) of the $86 \%$ ethanolic extract $(0.70 \pm 0.10 \mathrm{mg} \mathrm{BS} / \mathrm{g} \mathrm{DM})$, and Soxhlet 


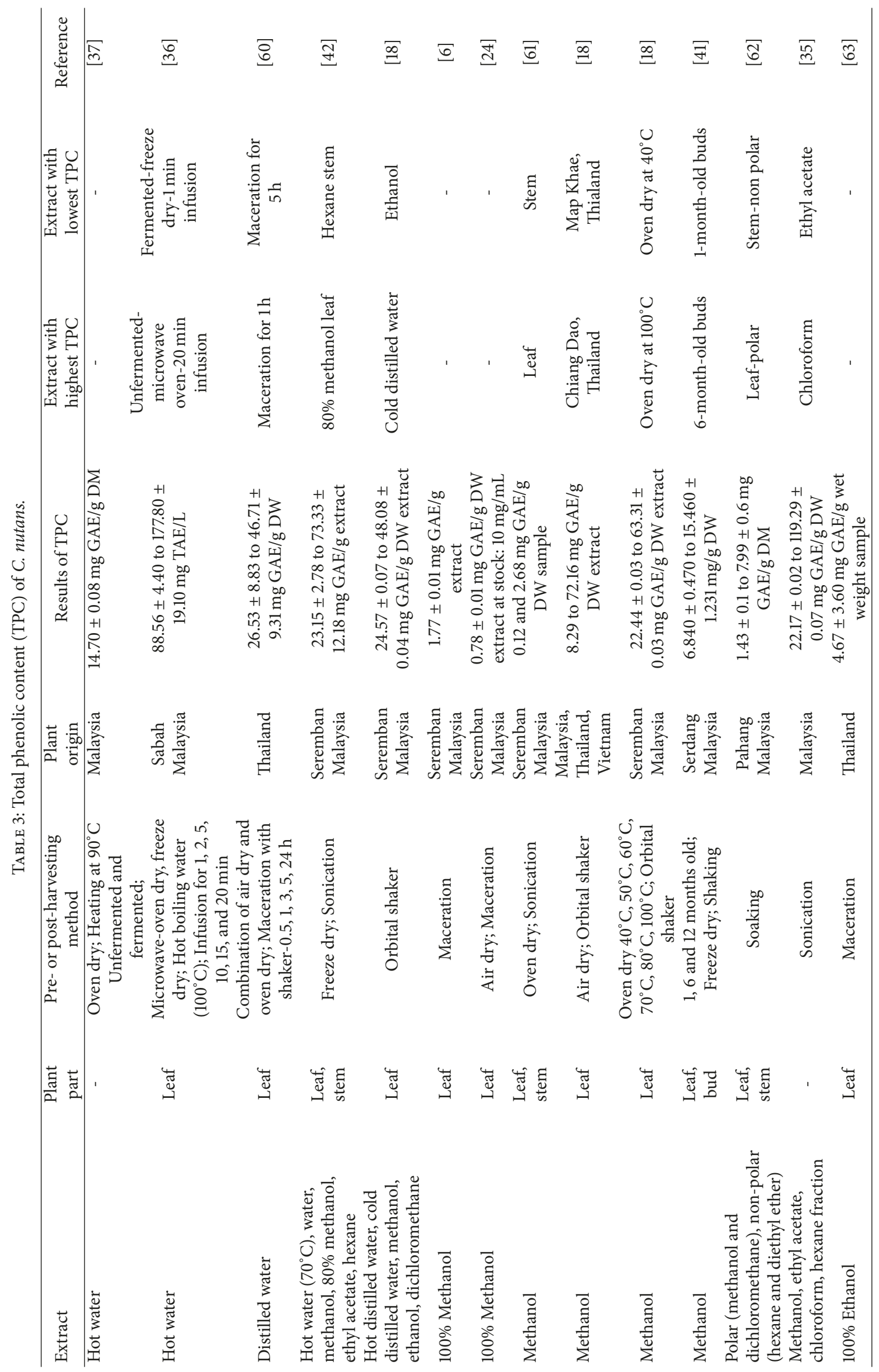




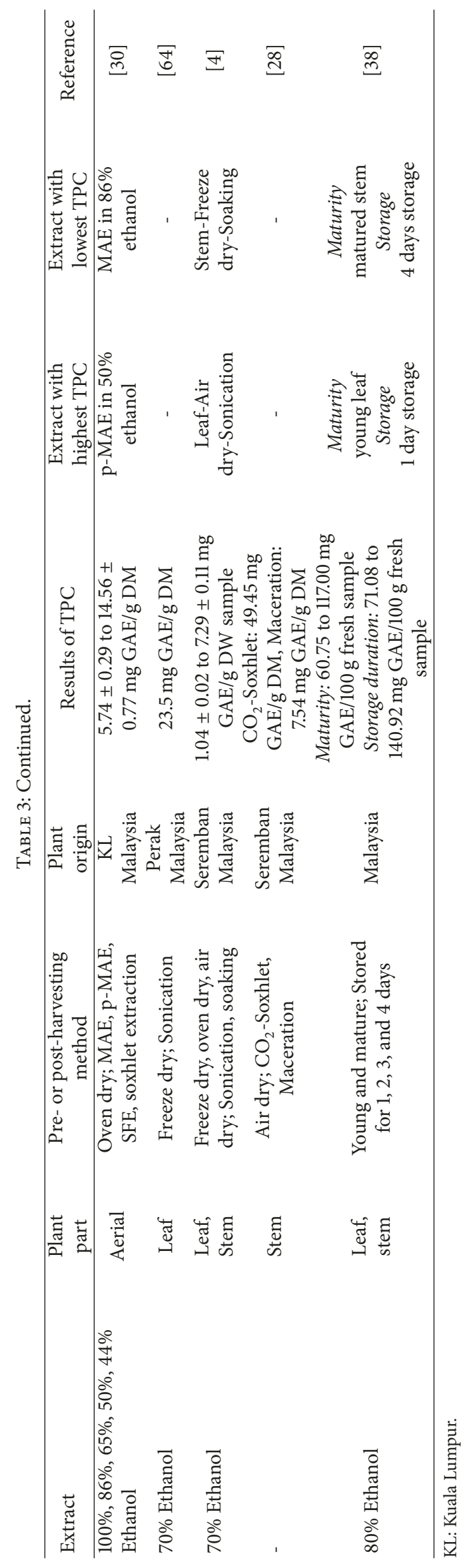




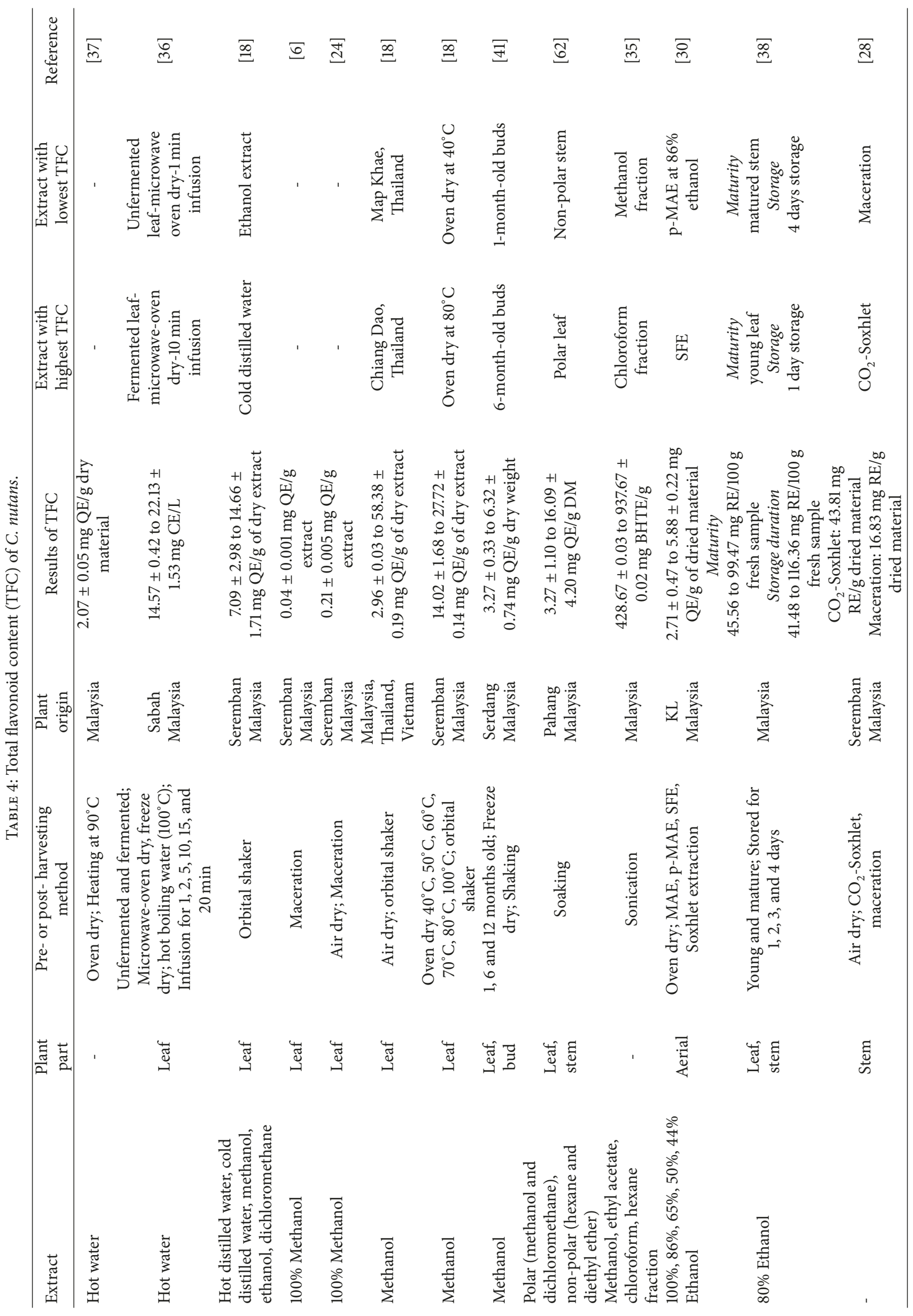


extraction of the absolute ethanolic extract $(0.47 \pm 0.20 \mathrm{mg}$ $\mathrm{BS} / \mathrm{g} \mathrm{DM})$. Additionally, the nutrient composition of $C$. nutans has also been determined by Sarega et al. [39] and Yu et al. [40] through proximate, vitamin, and mineral analysis as shown in Table 5.

Meanwhile, there were also studies that used HPLC for the quantification of phenolic compounds [23, 39, 41-43] and, in the case of Mustapa et al. [30], for the quantification of a phytosterol compound, $\beta$-sitosterol. The HPLC parameters used and the sample details are tabulated into Table 6. Although Wong et al. [43] attempted to quantify 4 phenolics compounds, only quercetin was found $(0.25$ $\pm 0.01 \mu \mathrm{g}$ per $\mathrm{mg}$ of dried sample), whereas Chelyn et al. [23] quantified schaftoside, orientin, isovitexin, and vitexin content for $C$. nutans leaves collected from 3 different sources. The results revealed that $C$. nutans collected from Taiping, Perak, Malaysia, possessed the highest amount of all 4 phenolics: $17.43 \pm 0.01 \mathrm{mmol} / \mathrm{g}$ of schaftoside, $0.86 \pm$ $0.04 \mathrm{mmol} / \mathrm{g}$ of orientin, $2.01 \pm 0.02 \mathrm{mmol} / \mathrm{g}$ of isovitexin, and $0.91 \pm 0.03 \mathrm{mmol} / \mathrm{g}$ of vitexin. Ghasemzadeh et al. [41] found that samples with different maturity and from different plant parts retained different phenolics and flavonoids. Six-monthold bud extract was found to be able to retain the highest concentration of gallic acid (5.96 $\pm 0.55 \mathrm{mg} / \mathrm{g} \mathrm{DW})$. Sarega et al. $[39,42]$ analyzed and quantified a total of 8 phenolics in the extract. Both studies found that protocatechuic acid was the most prominent phenolic acid in the hot water, water, and $80 \%$ methanol leaf extracts $(33.28 \pm 0.01 \mathrm{mg} / \mathrm{g}$ extract $)$. Finally, aerial extract that underwent SFE possessed the highest amount of $\beta$-sitosterol $(0.83 \pm 0.10 \mathrm{mg} / \mathrm{g} \mathrm{DM})$ followed by the $86 \%$ ethanolic p-MAE extract $(0.65 \pm 0.14 \mathrm{mg} / \mathrm{g} \mathrm{DM})$, $86 \%$ ethanolic MAE extract $(0.52 \pm 0.10 \mathrm{mg} / \mathrm{g} \mathrm{DM})$, and Soxhlet assisted extract $(0.23 \pm 0.18 \mathrm{mg} / \mathrm{g} \mathrm{DM})$ [30].

\section{Pharmacological Activity}

3.1. Pharmacological Activity of C. nutans Extracts and Fractions. A number of $C$. nutans' traditional uses have been further verified by laboratory experiments. They have been categorized into antivenom, analgesic, anti-inflammatory, immunomodulating, neuroprotective and neuromodulating function, antidiabetic and $\alpha$-glucosidase inhibitory, antioxidant, antiviral, antibacterial, antifungal, anticancer, wound healing, plasmid DNA protective, lipid elevated inhibition, and oral mucositis and stomatitis treatment activities. Tables $7(\mathrm{a}-\mathrm{o})$ summarize all the available data regarding the pharmacological activities of $C$. nutans extracts and fractions accordingly. All the tables were systematized based on experimental model, followed by type of assay and polarity of extract used. Generally, diverse results can be observed for the same pharmacological activity. The extract preparation method, origin, concentration, and assays type are the primary factors that contributed to the variation in results, and a short summary and point of view have been included in each section.

3.1.1. Antivenom Activity. Table 7(a) summarizes the antivenom tests that have been carried out thus far on $C$. nutans.
The experiments examined the antivenom properties of $C$. nutans' leaves against snake, scorpion, and bee venoms. There were a total of 2 in vitro and 4 in vivo antivenom studies, and the extracts tested were prepared from water, ethanol, and aqueous ethanol. From the results of the in vitro studies, the water extract at $0.706 \mathrm{mg} / \mathrm{mL}$ exhibited a moderate antiscorpion venom effect through a direct inactivation mechanism [65] while the aqueous ethanol extracts were ineffective against bee venom [66]. On the other hand, in 3 out of the 4 in vivo studies conducted, the extracts ( 2 water and 1 aqueous ethanol extracts) notably did not exhibit antivenom effects after the test subjects were given different doses (6 to $2000 \mathrm{mg} / \mathrm{kg} \mathrm{bw}$ ) of the extracts through intraperitoneal injection (i.p.), intervenous injection (i.v.), or per os (p.o.) [5, $7,67]$. Although there was 1 in vivo study proposing that the water extract of $C$. nutans could exhibit a moderate anti-snake venom effect, the study did not include the administration method or the doses given [67]. Thus, defining a suitable extract that is biologically active as an antidote for snake and insect venoms is ambiguous. In summary, among the extracts tested, only water leaf extract exhibited potential in vitro inhibition effect towards snake and insect venoms via direct inactivation mechanism. Further animal studies that focus on studying the metabolites of water extract and their reaction with the mediators that responsible for the direct inactivation pathway are worth emphasizing. Clinical study that investigates the efficacy of water extract as an antivenom agent through application of topical formulation on the patients might lead to a possible breakthrough.

3.1.2. Analgesic Activity. Table 7(b) displays the scientific studies that investigated the analgesic ability of C. nutans through 3 different types of in vivo assays: the acetic acidinduced writhing test, formalin-induced paw licking test, and hot plate test. Three studies have used the acetic acidinduced writhing test to examine the analgesic effect of C. nutans extracts prepared using 4 different types of solvents $[19,68,69]$. The results suggested that treating mice with the $n$-butanol extract at $90 \mathrm{mg} / \mathrm{kg}$ as well as with the methanol extract at $279.3 \mathrm{mg} / \mathrm{kg}$ was as potent in terms of analgesic properties as treating them with phenylbutazone at $100 \mathrm{mg} / \mathrm{kg}$. On the other hand, the study by Abdul Rahim et al. [19] was the only one that used formalin-induced paw licking test to evaluate the analgesic properties of C. nutans. The results suggested that the methanol extract, with a half maximal effective concentration $\left(\mathrm{EC}_{50}\right)$ at $227.7 \mathrm{mg} / \mathrm{kg}$, was able to relieve pain in the late phase (centrally inflammatory induced pain pathway) via opioid/nitric oxide- (NO) mediated, but cyclic guanosine monophosphate- (cGMP-) independent modulation systems. In addition, the analgesic effects of the absolute methanol, ethanol, and $n$-butanol extracts have been examined through the hot plate test. The results suggested that $500 \mathrm{mg} / \mathrm{kg}$ methanol extract was effective in alleviating the pain response at the interval from 60 to $210 \mathrm{~min}$ while both the ethanol and butanol extracts showed no analgesic effects up to $5 \mathrm{~g} / \mathrm{kg}$. Overall, methanol extract of $C$. nutans exerted potential analgesic activity in both acute and persistent pain tests. The study also suggested 


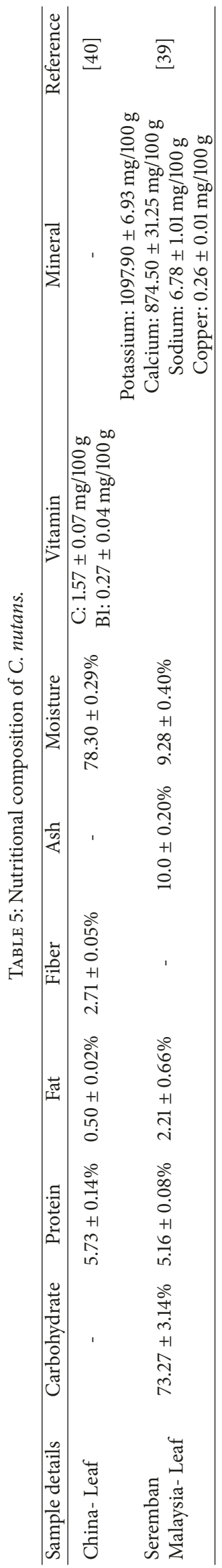




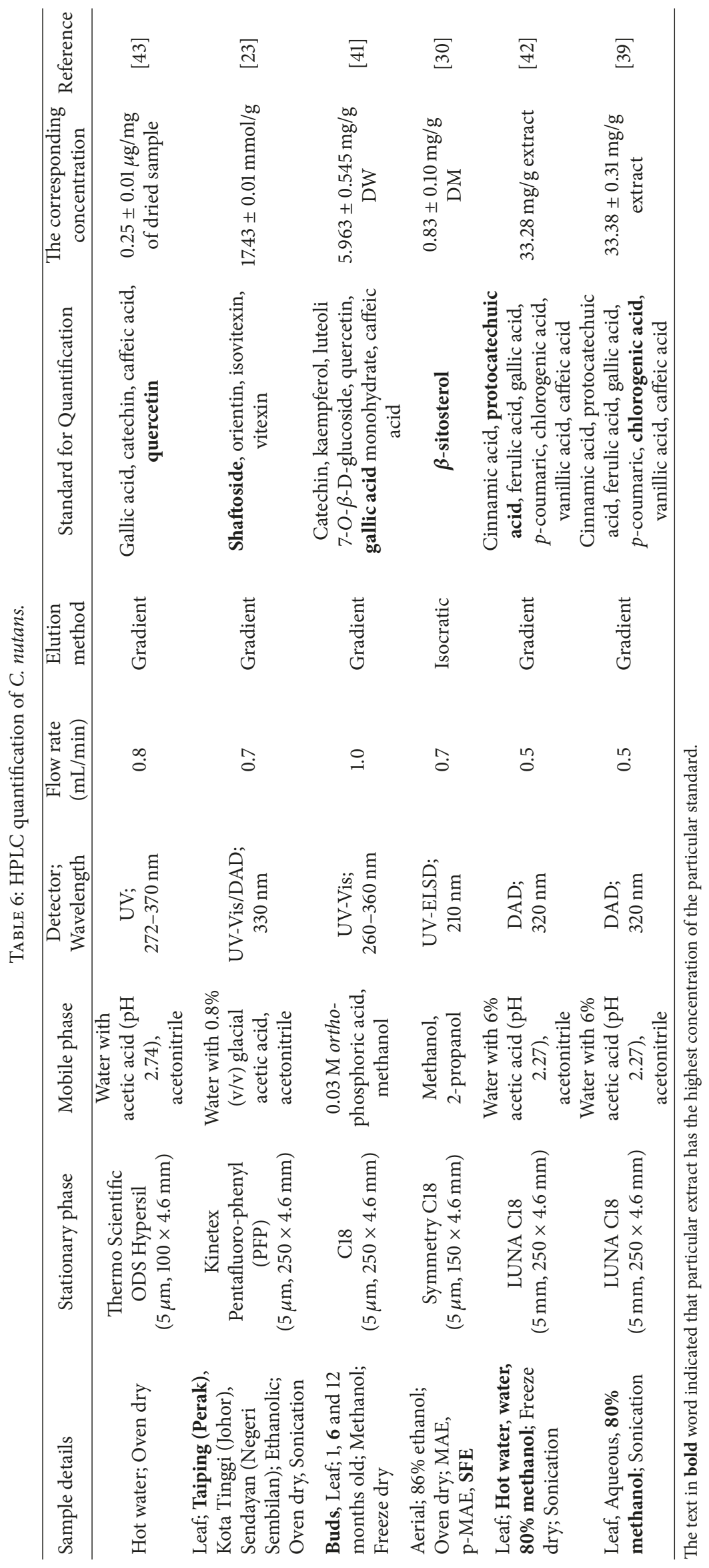




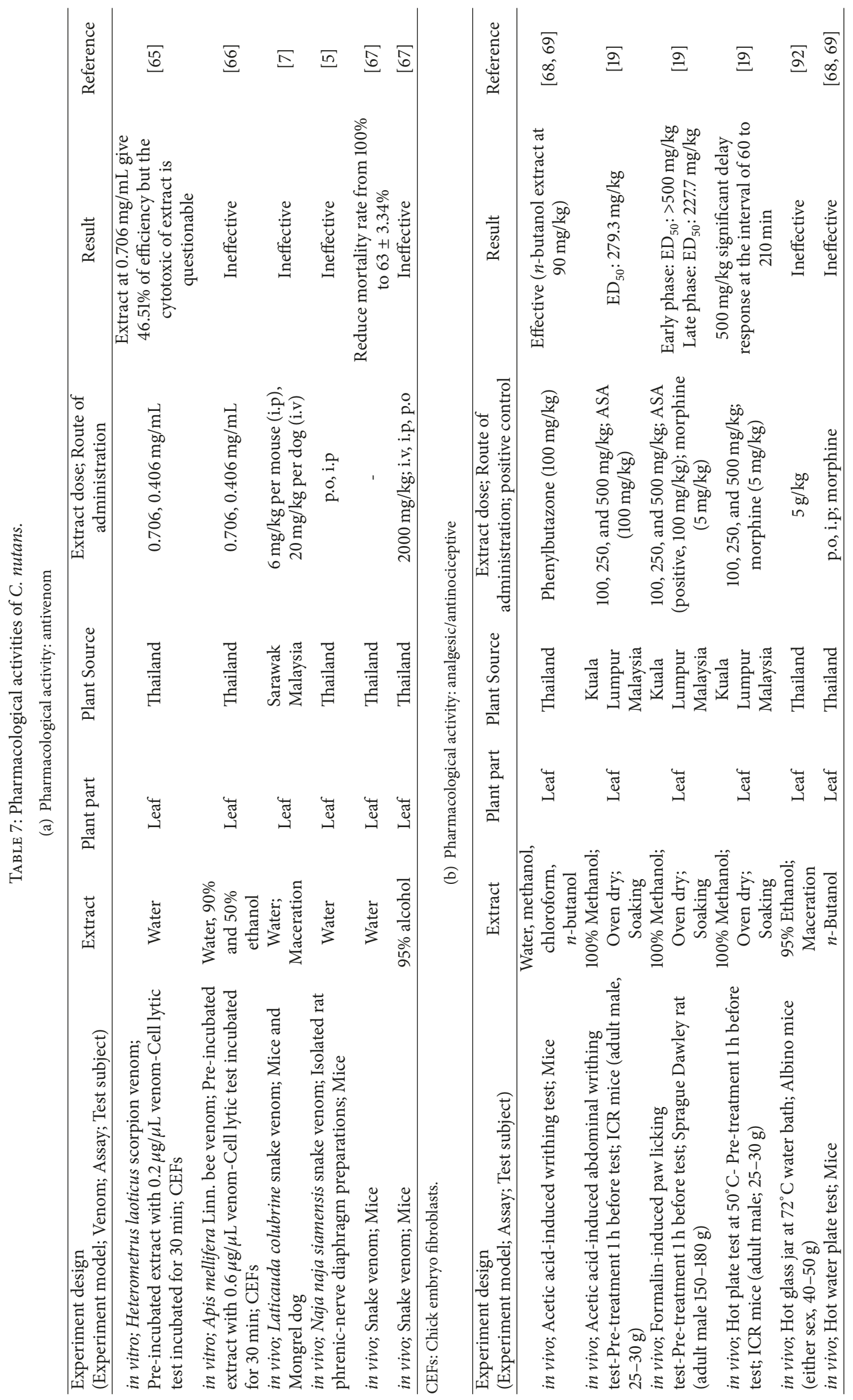




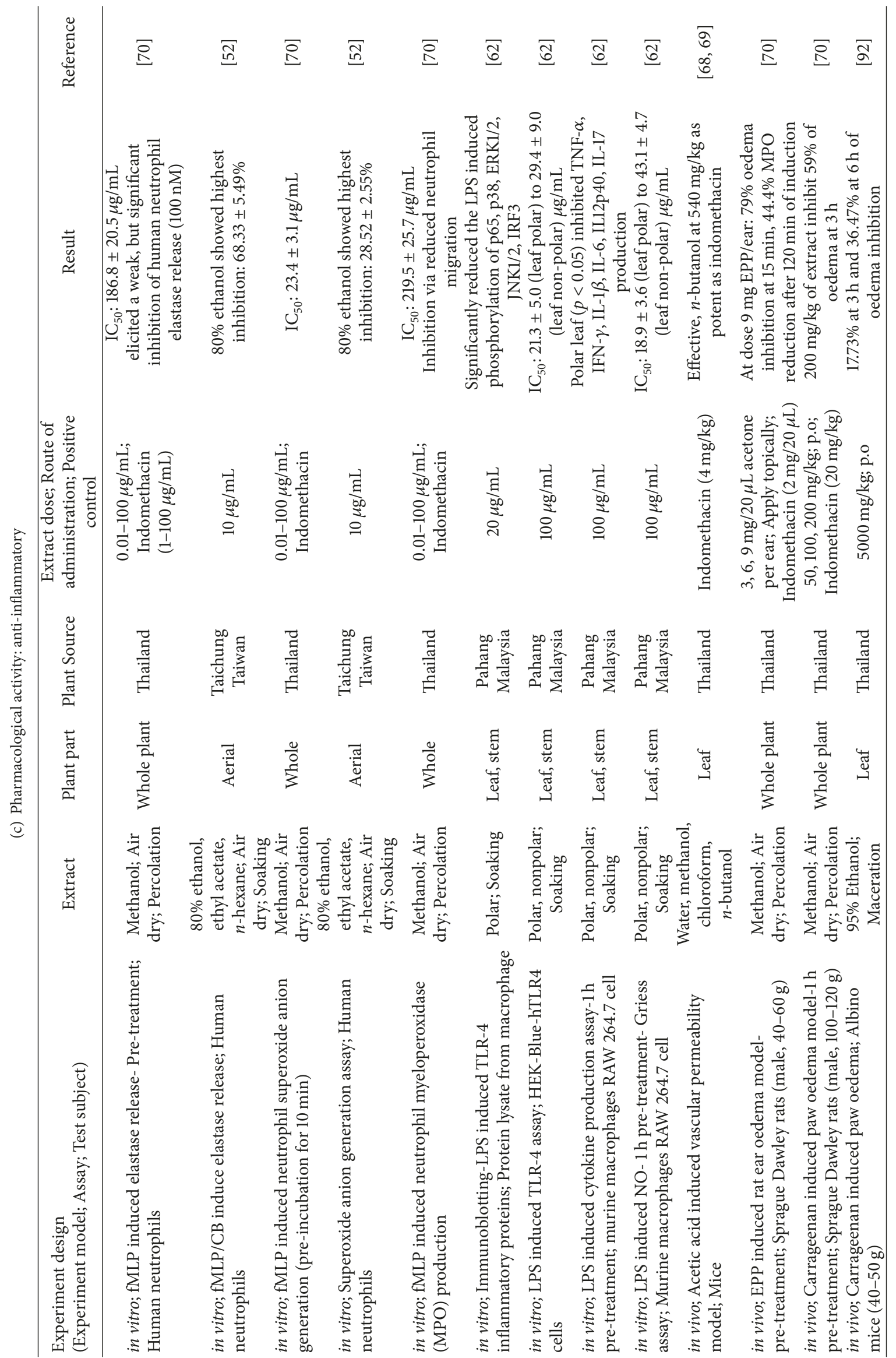




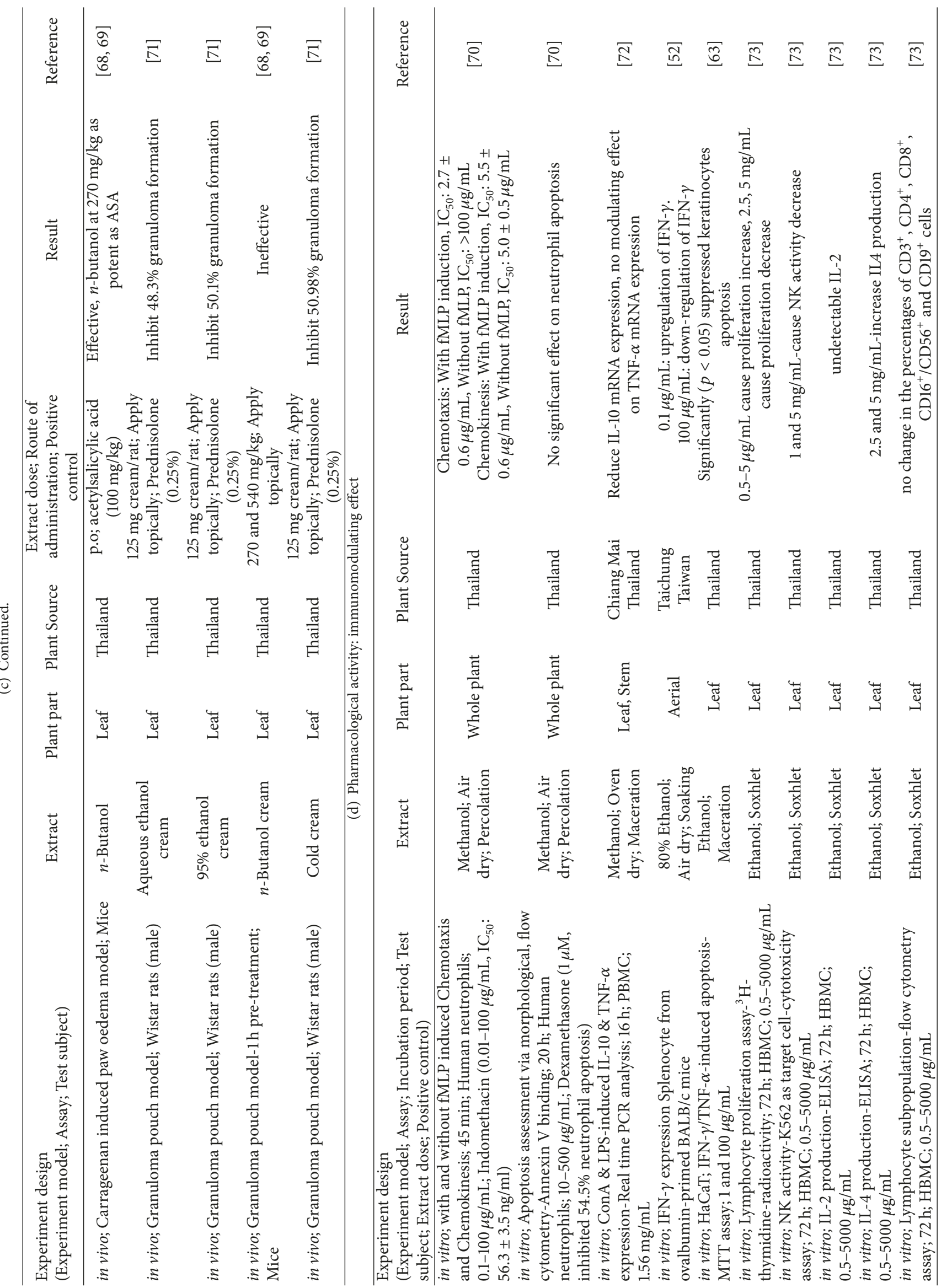




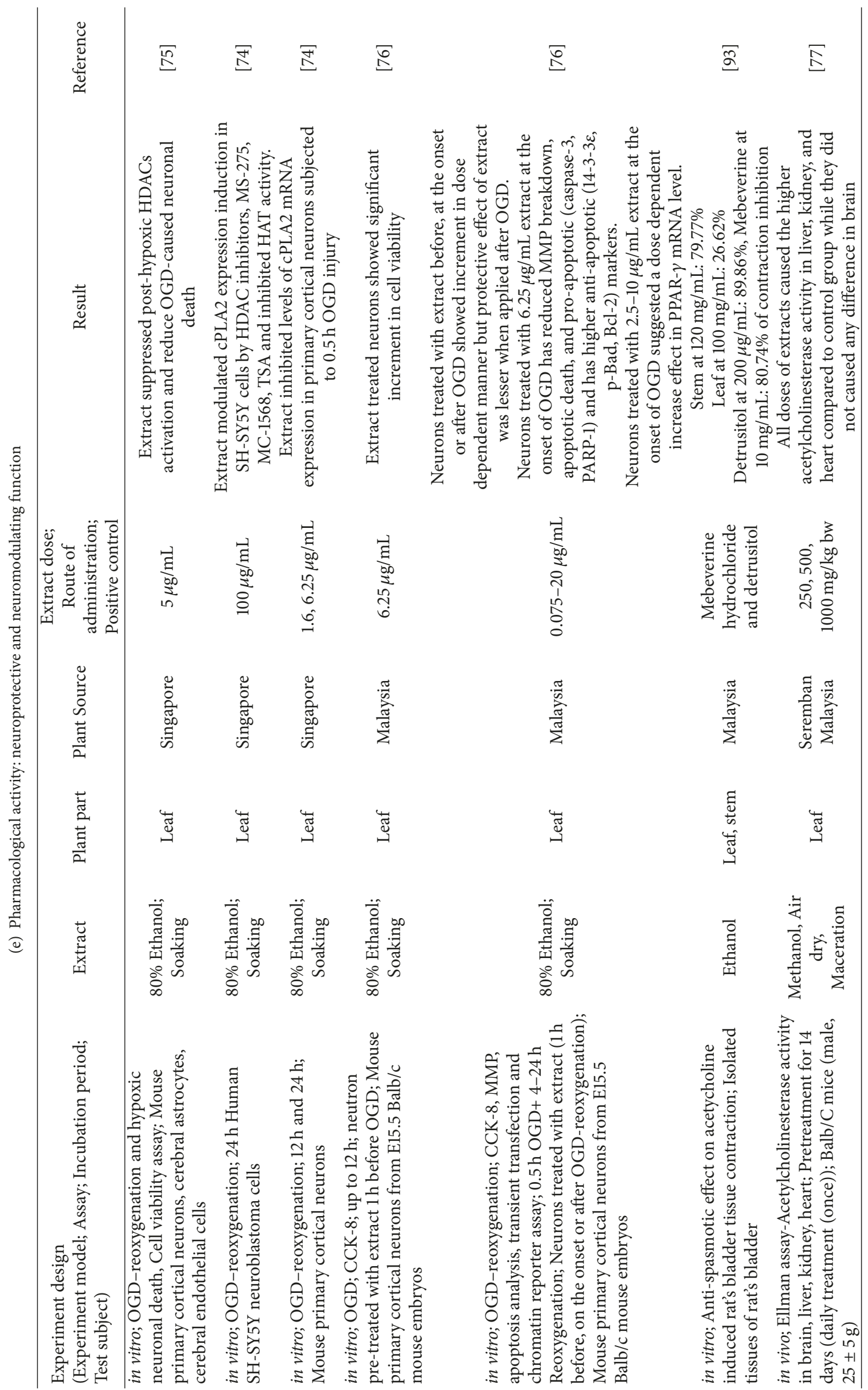




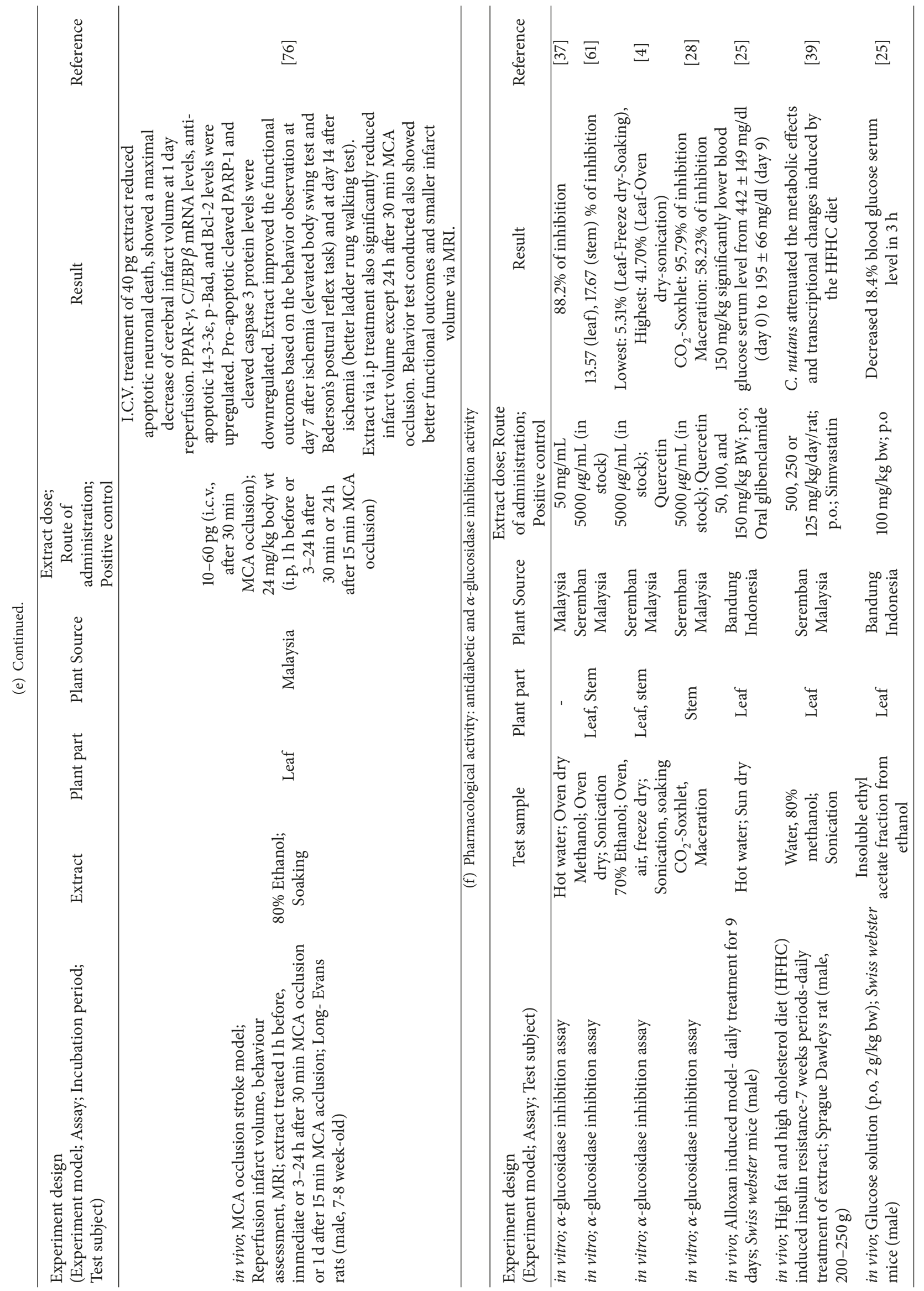




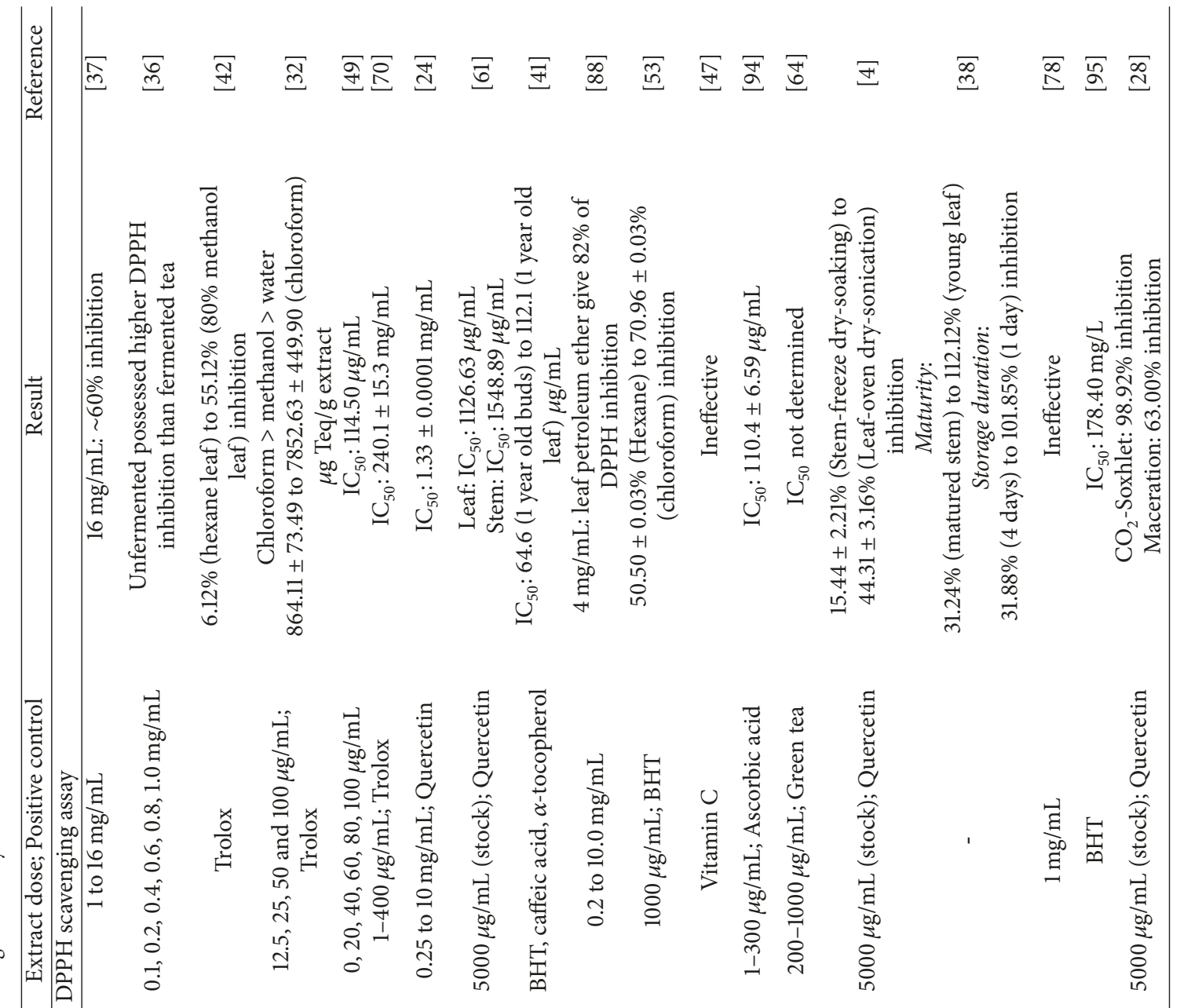

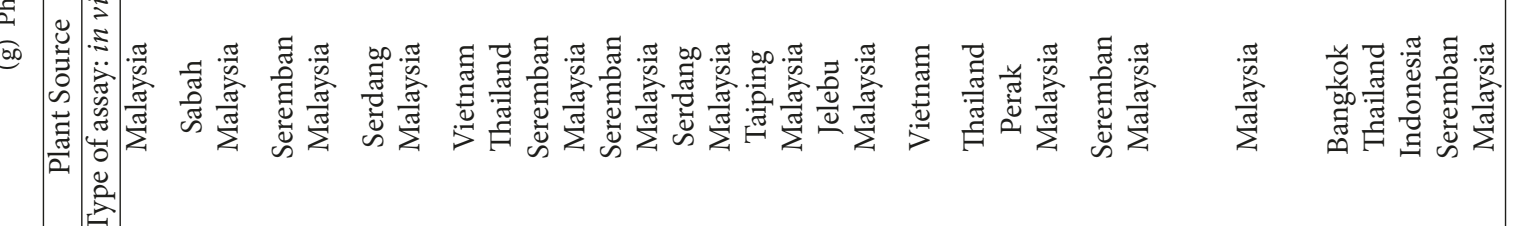

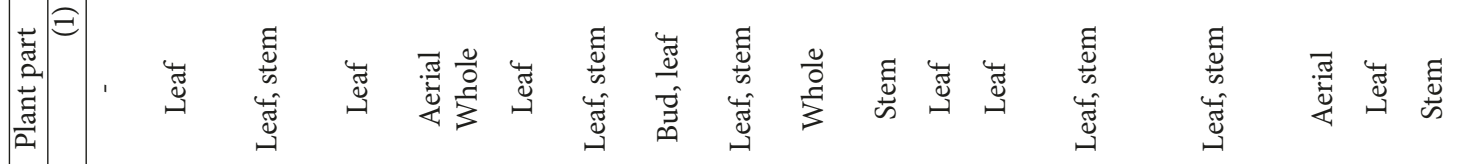

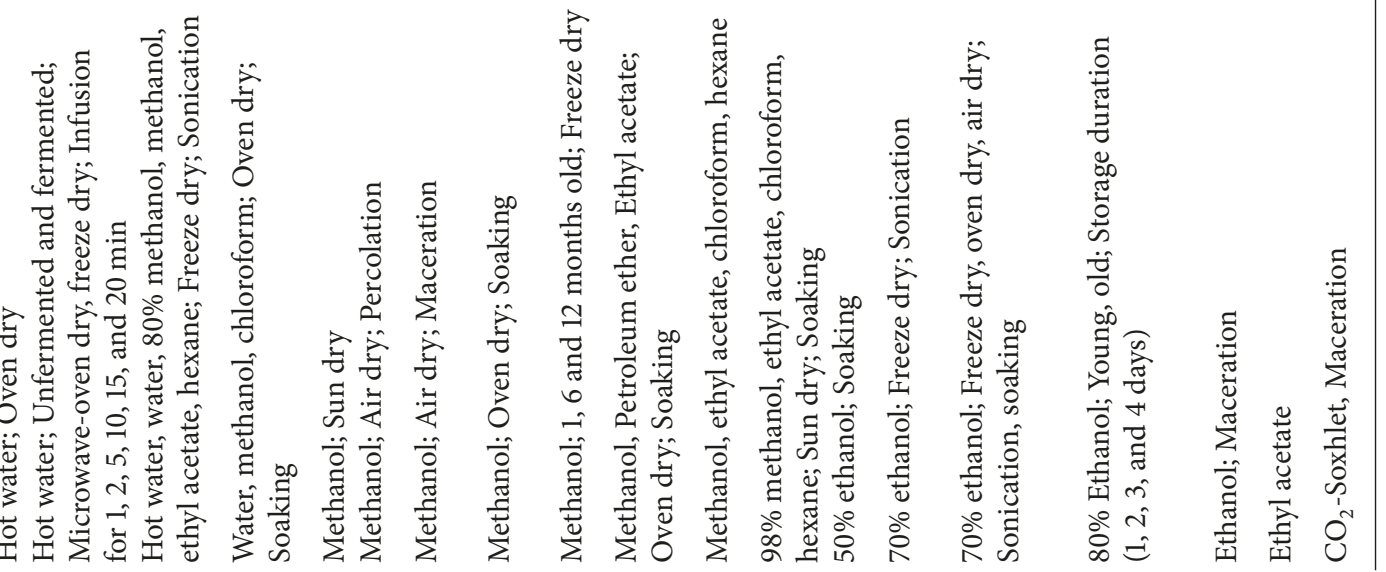




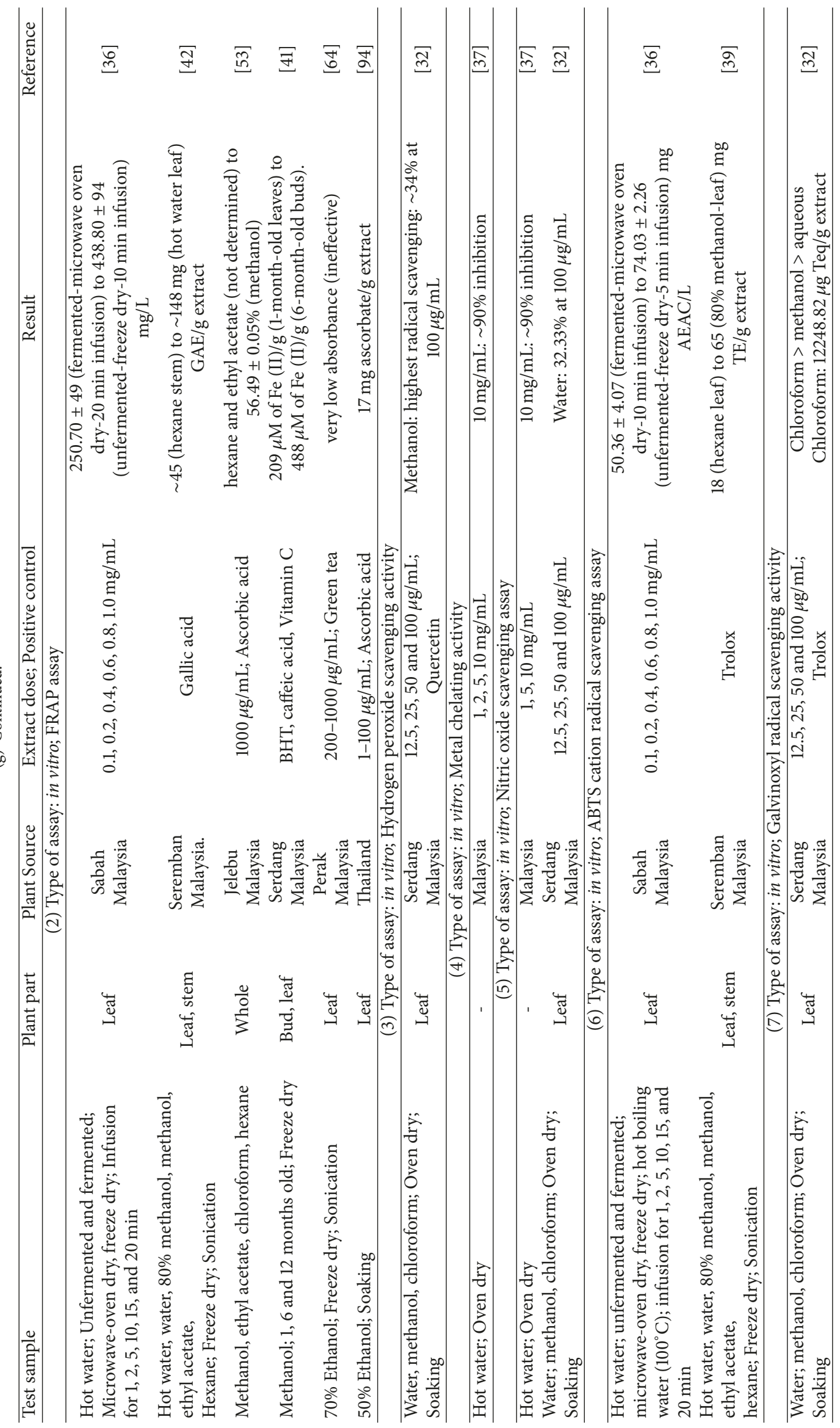




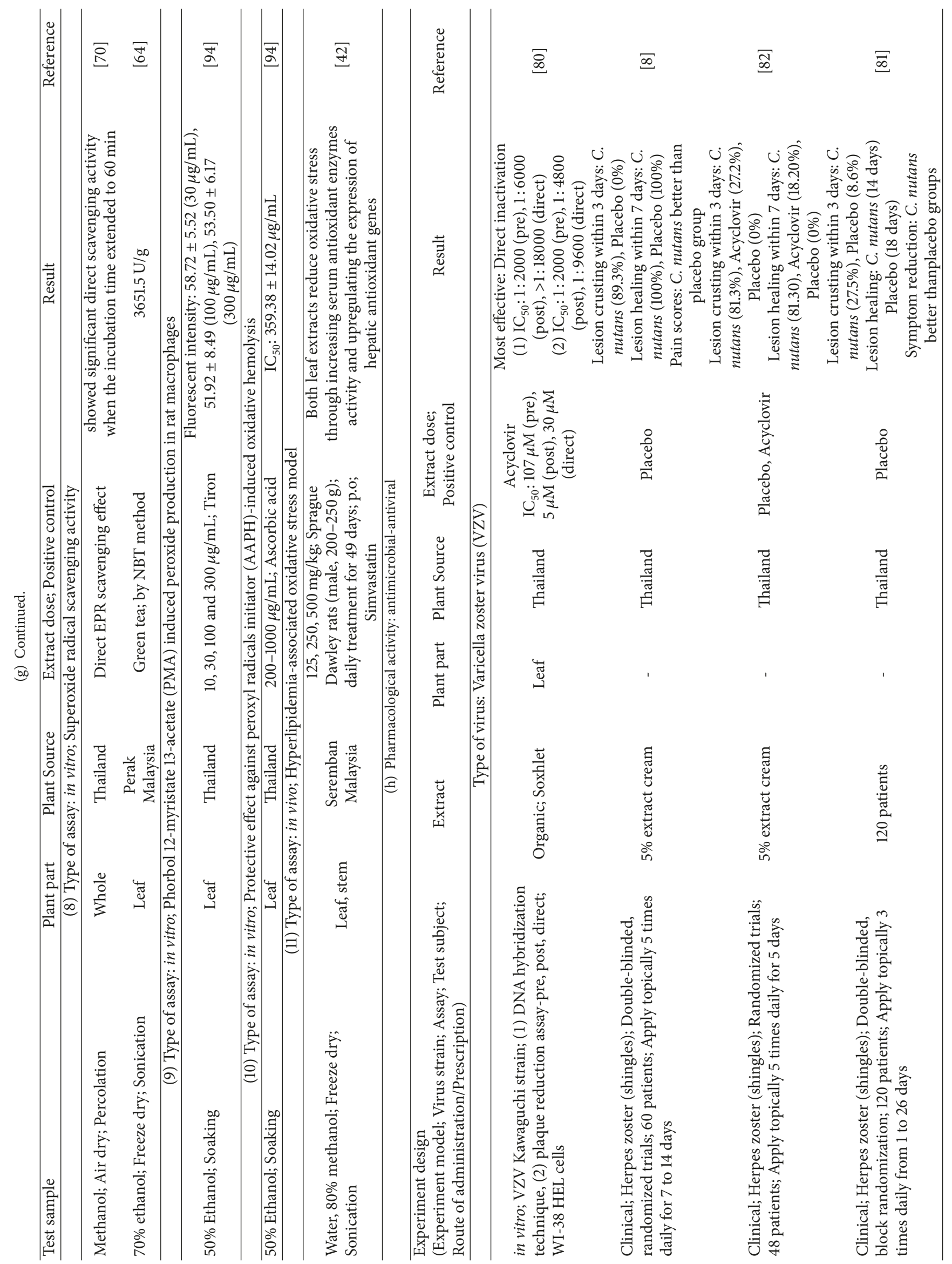




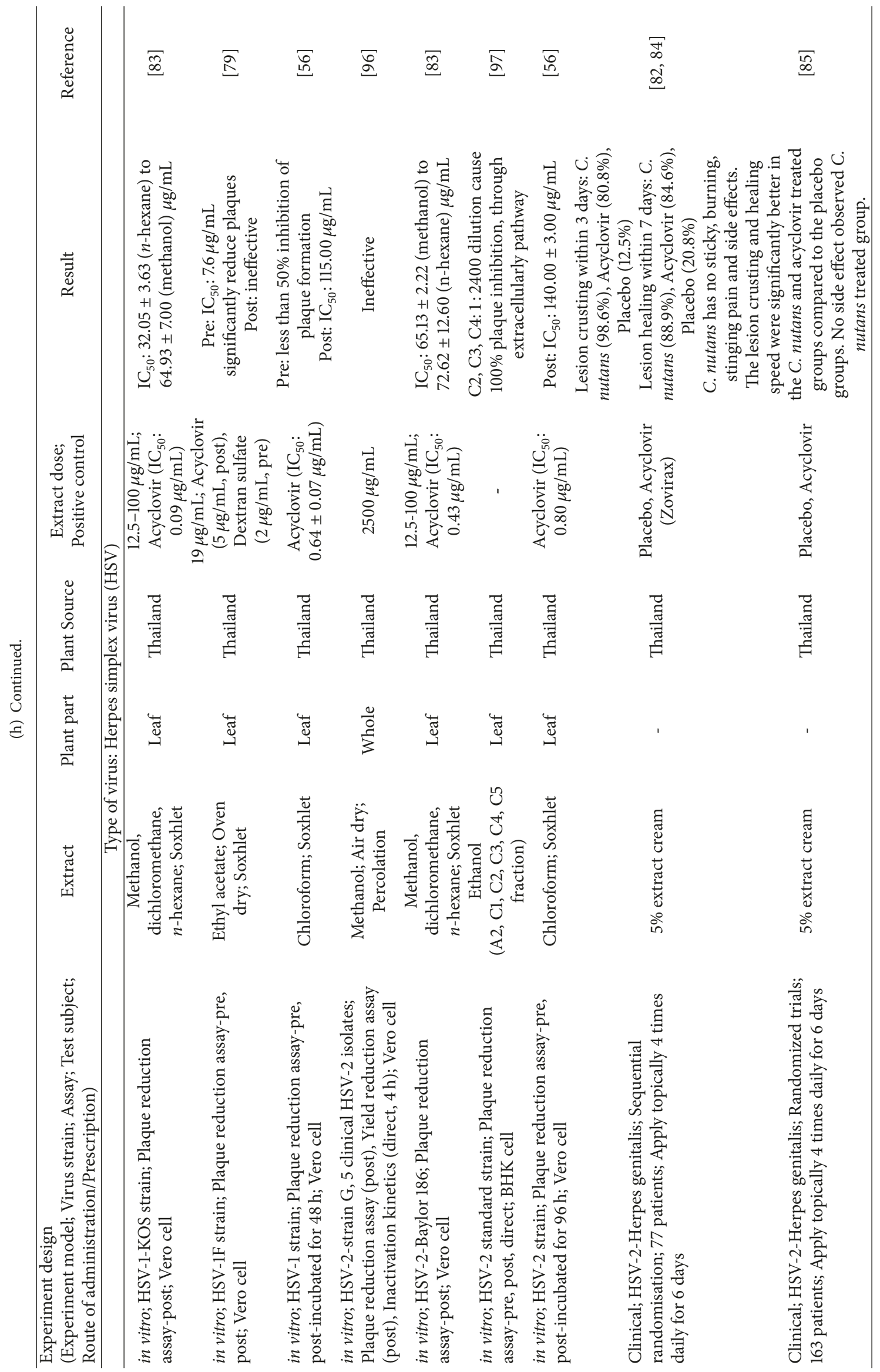




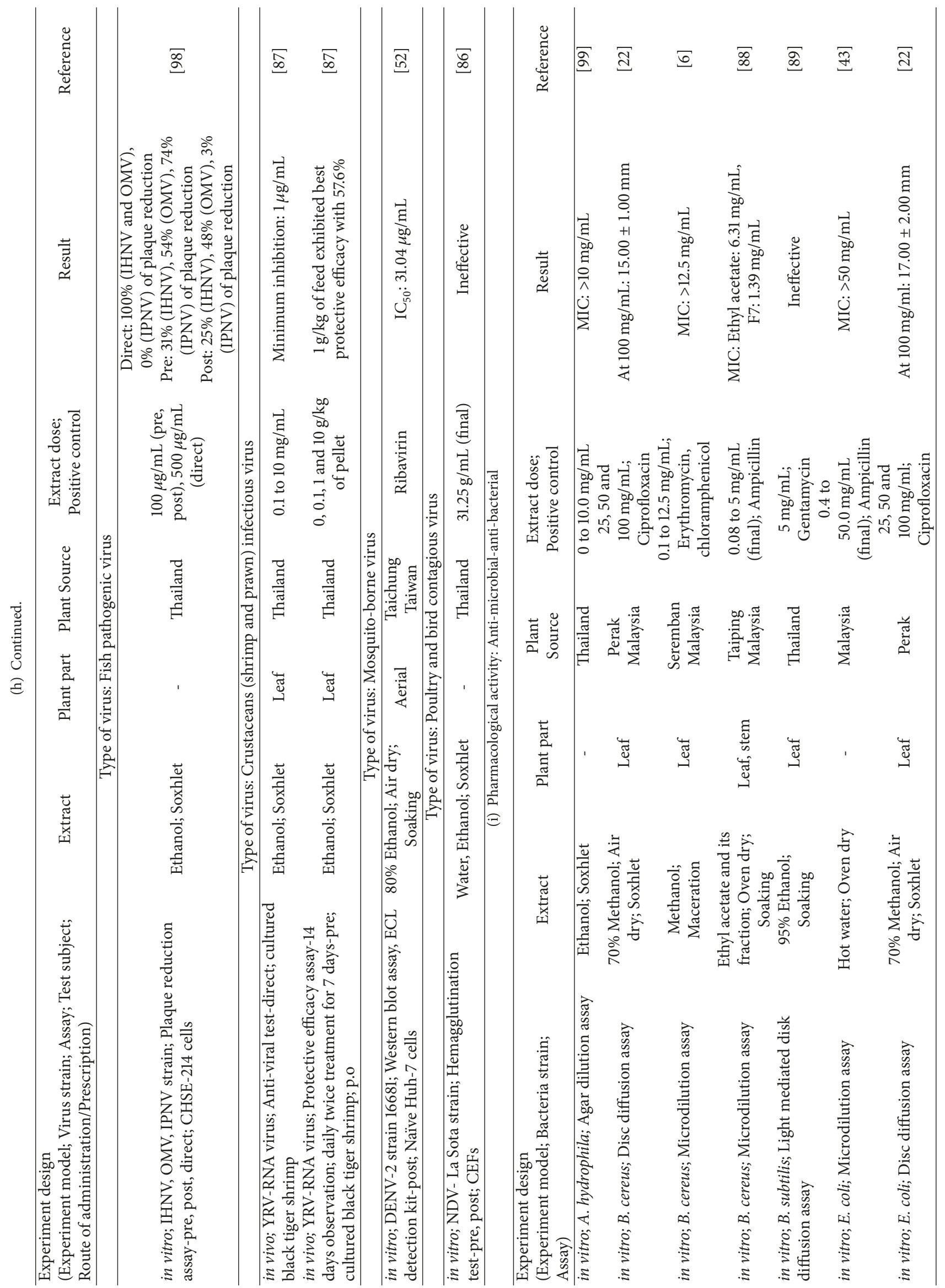




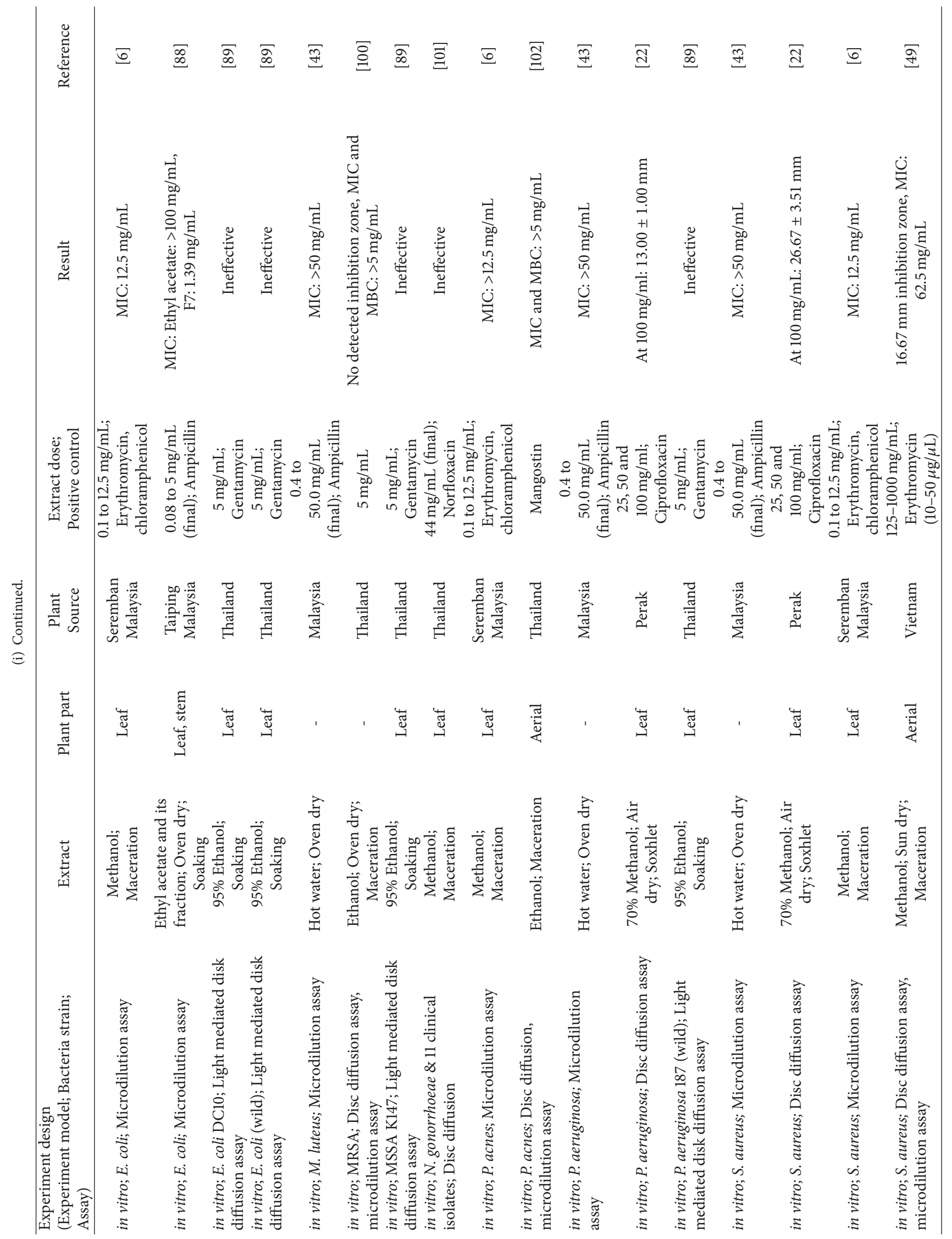




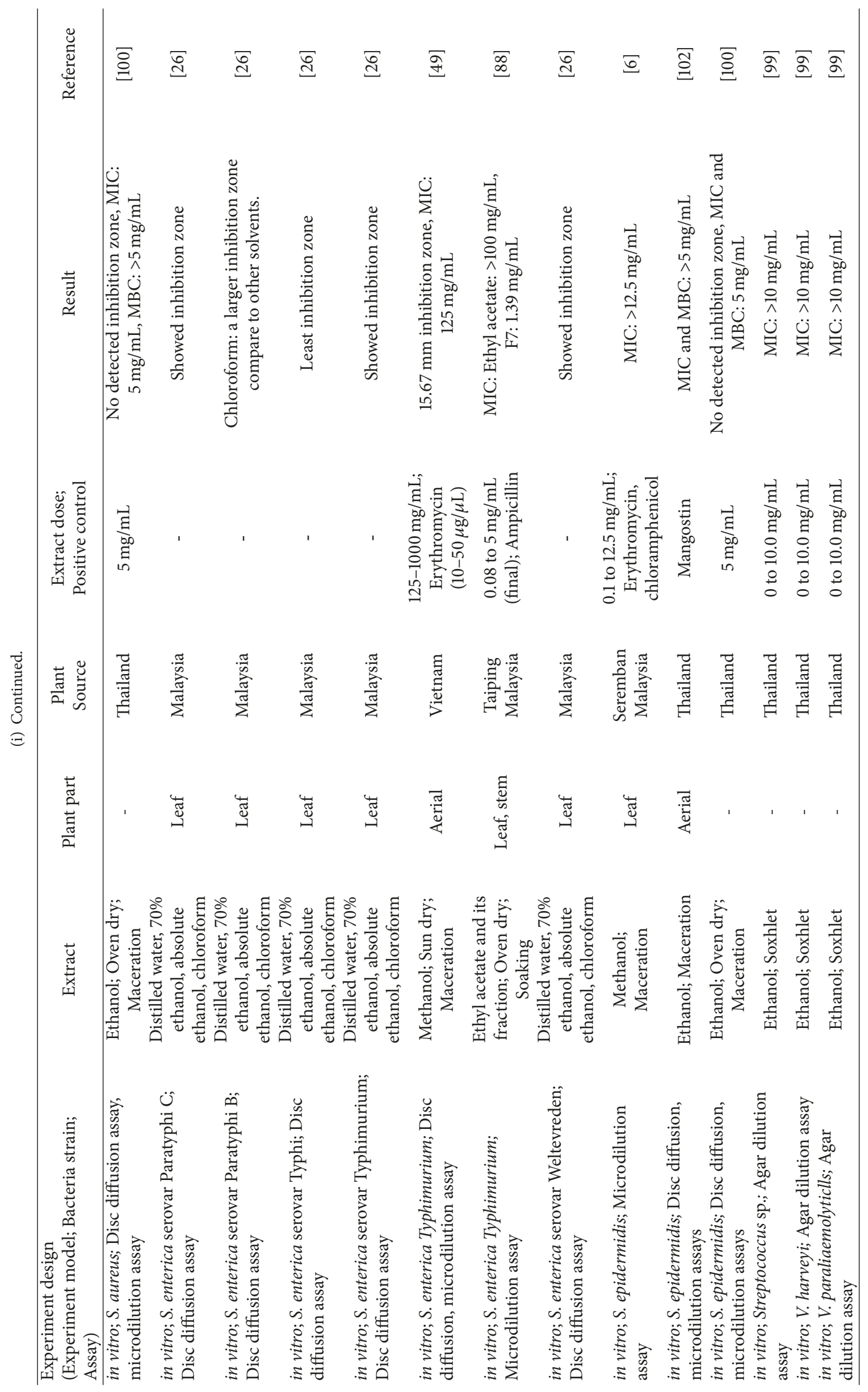




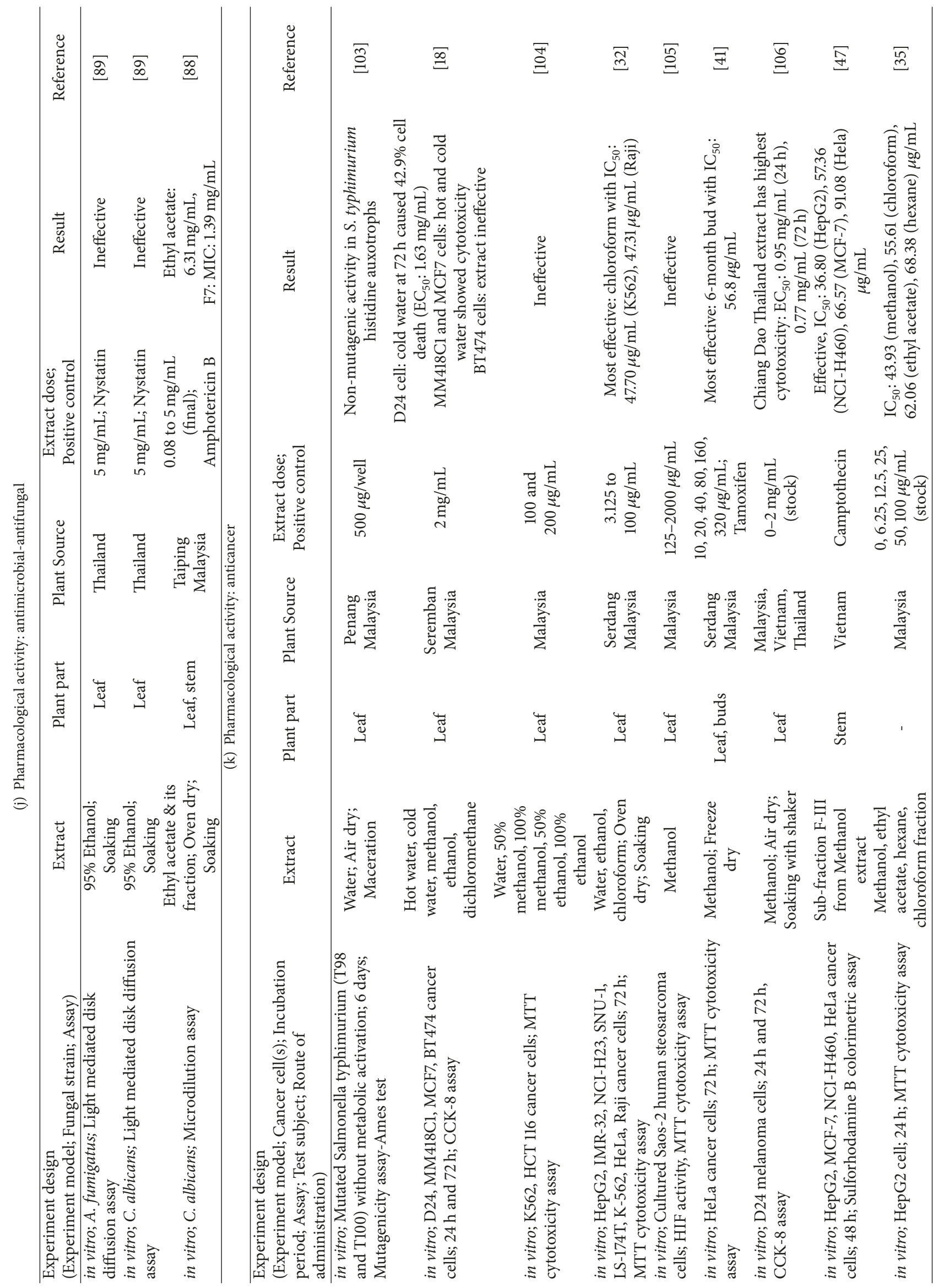




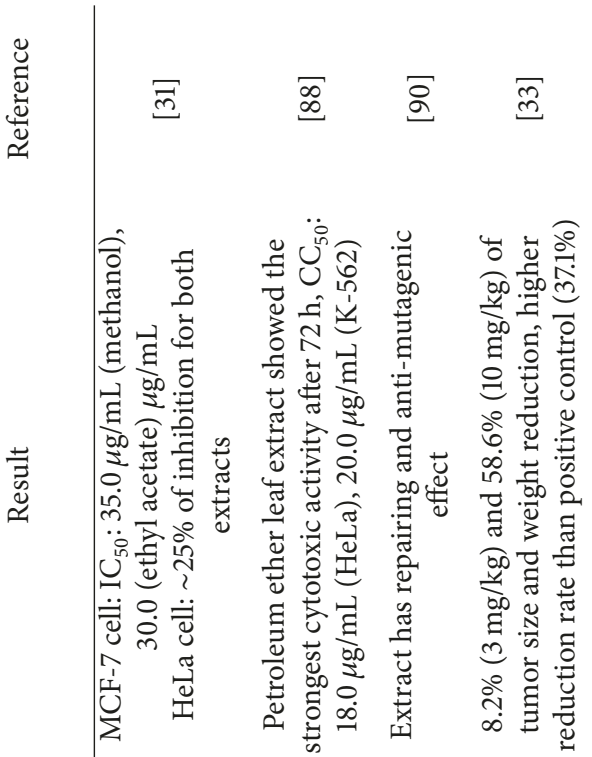

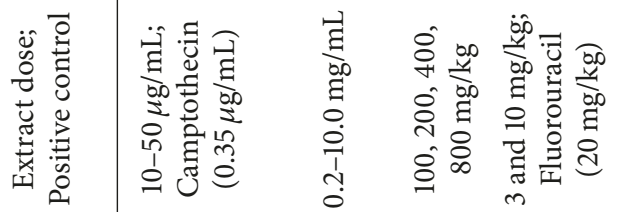

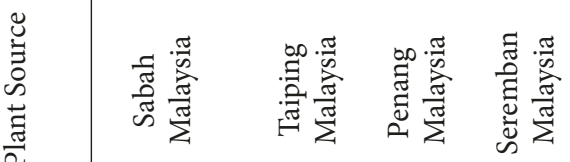

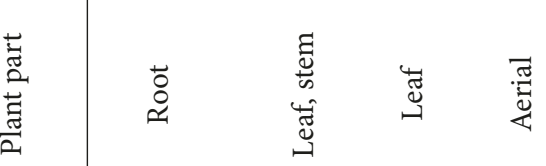

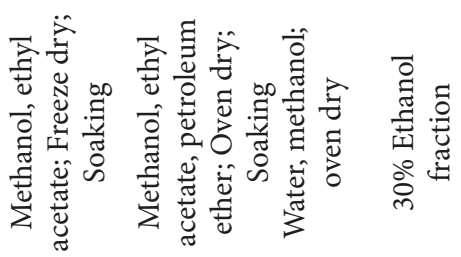

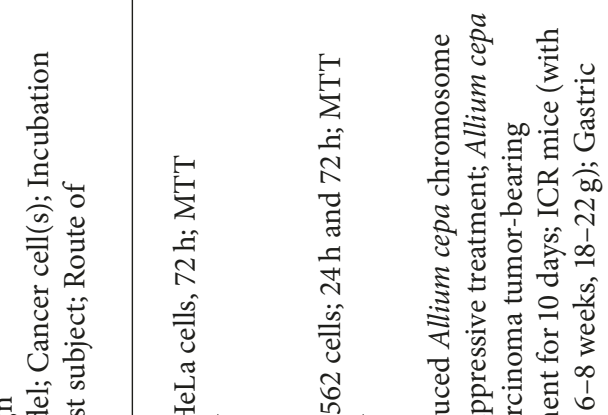

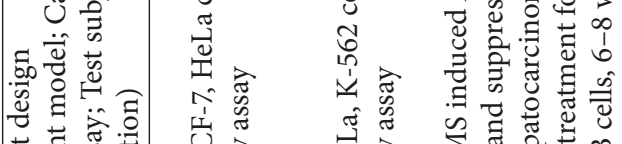

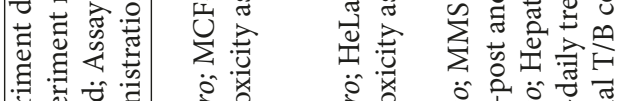

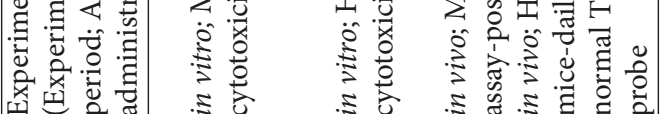
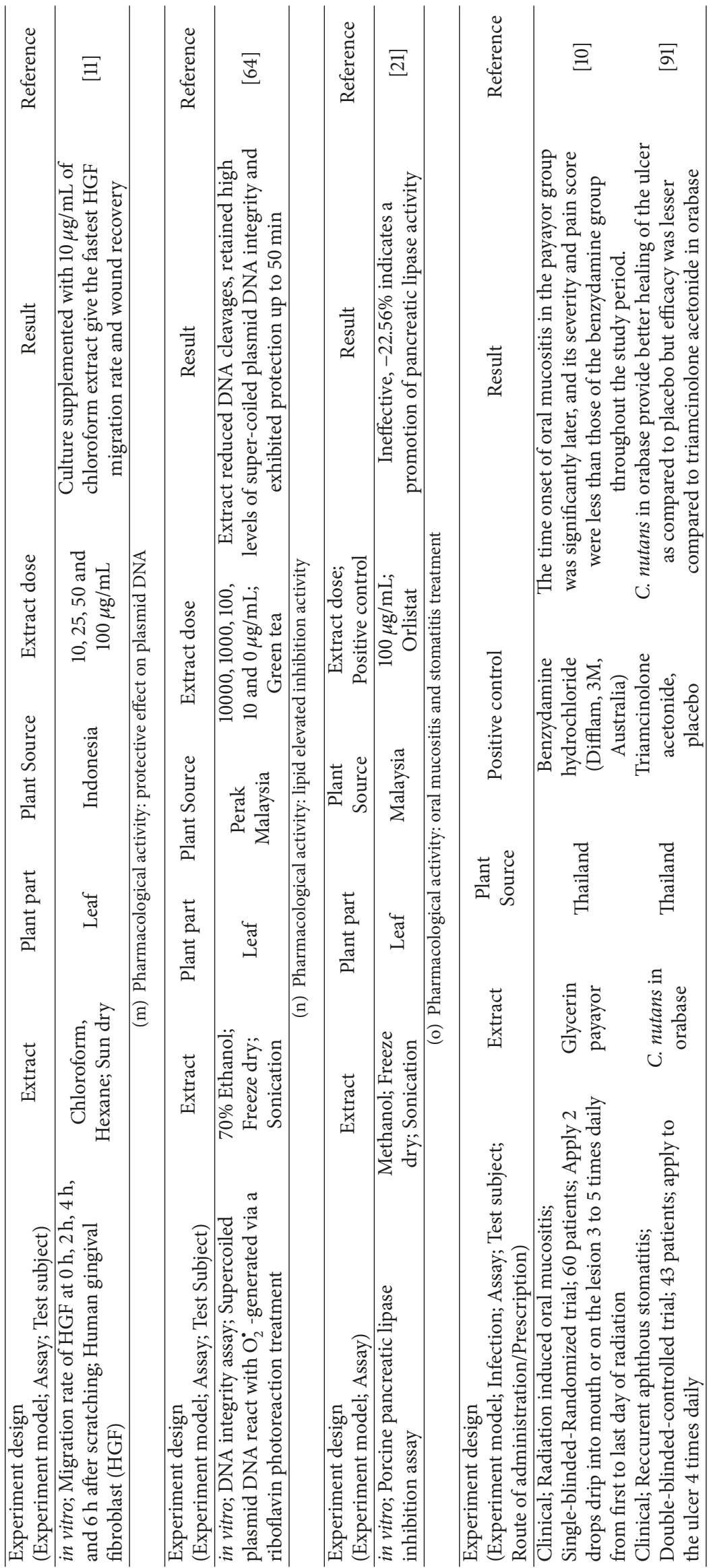

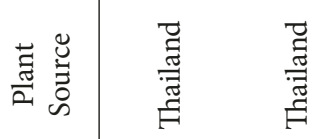

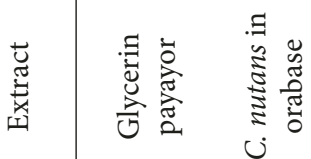

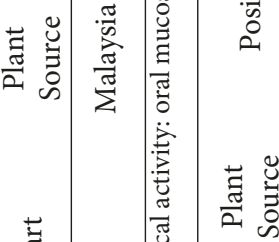

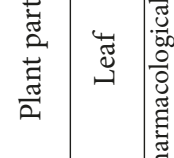

코
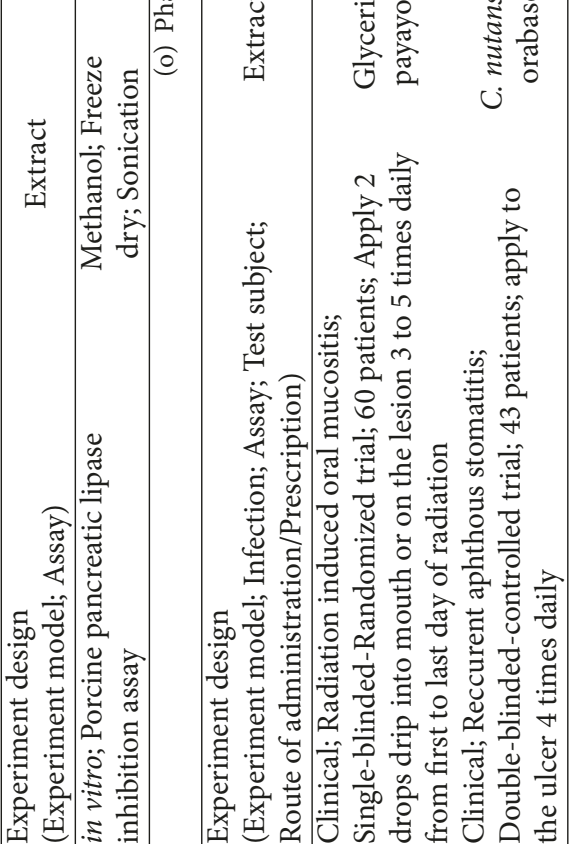


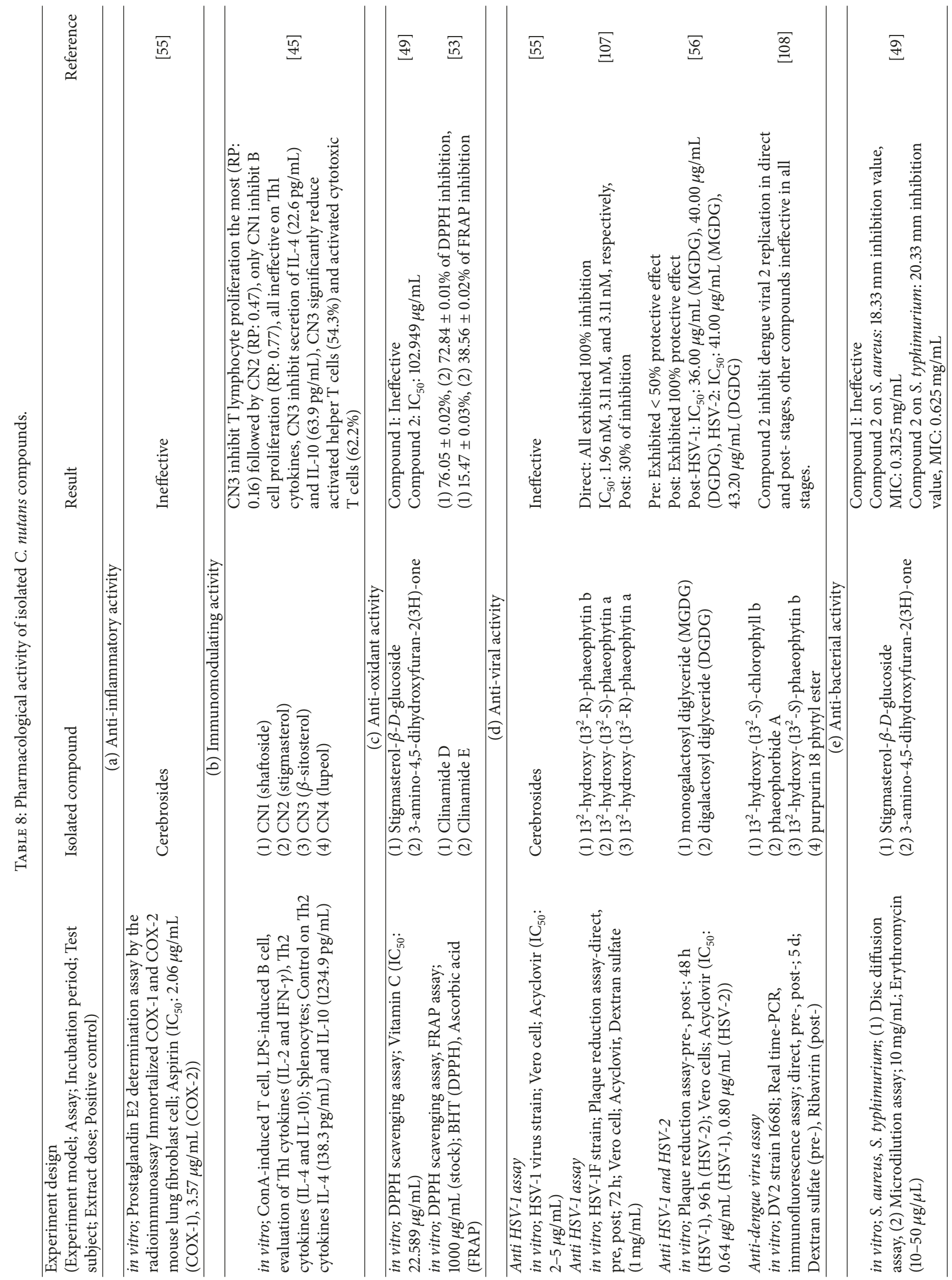




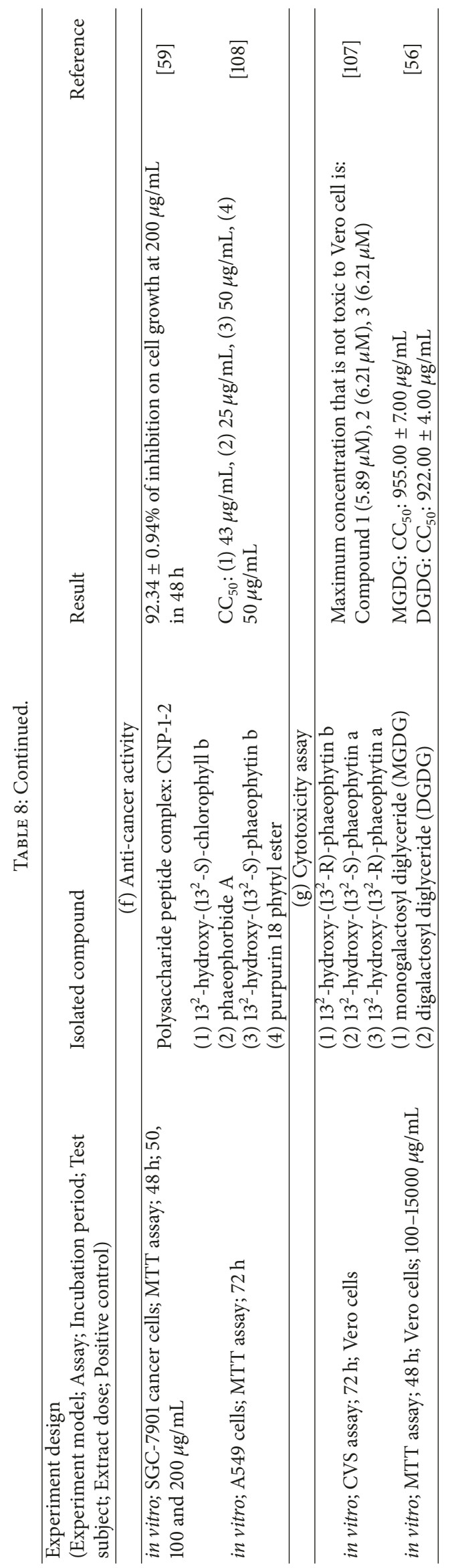


that the presence of phenolics particularly gallic acid, caffeic acid, ferulic acid, vitexin, and apigenin which previously reported to exert antinociceptive activity which might be the main activity contributors. Since methanol extract might be a potential analgesic agent, it is important to further investigate the efficacy dosages, sites of action, and the level of the extract to modulate the pain along the pathway suggested. Furthermore, study may also provide information regarding antinociception properties of $C$. nutans methanol extract on visceral and neuropathic pains.

3.1.3. Anti-Inflammatory Activity. Table 7(c) summarizes the anti-inflammatory effect of $C$. nutans. The anti-inflammatory effect of $C$. nutans was assessed based on in vitro assays, such as the macrophage activator $N$-formyl-methionylleucyl-phenylalanine- (fMLP-) induced neutrophil elastase release superoxide anion generation, lipopolysaccharides (LPS) induced toll like receptor 4 (TLR-4), NO Griess, and cytokine production assays. Furthermore, the types of in vivo experiments used to evaluate the acute anti-inflammatory properties of $C$. nutans were the acetic acid-induced vascular permeability model and ethyl phenylpropiolate- (EPP) induced rat ear oedema and carrageenan-induced paw oedema model, whereas its subchronic anti-inflammatory effect was assessed using the granuloma pouch model, which mimics subchronic inflammation in humans. For the in vitro anti-inflammatory effects, both the methanol and $80 \%$ ethanol extracts have been evaluated through the neutrophil elastase release and superoxide anion generation assays [52, 70]. The results showed that $10 \mu \mathrm{g} / \mathrm{mL}$ of $80 \%$ ethanol extract inhibited $68.33 \%$ elastase release, which was more effective than the methanol extract $(<20 \%)$, while to inhibit superoxide radical formation, both extracts at $10 \mu \mathrm{g} / \mathrm{mL}$ showed comparable efficacy, as they inhibited approximately $30 \%$ superoxide formation [52, 70]. For the LPS-induced TLR-4, $\mathrm{NO}$, and cytokine production assays, the results suggested that the polar (methanol and dichloromethane) extract possessed a better $\mathrm{IC}_{50}$ value $(<22 \mu \mathrm{g} / \mathrm{mL})$ for inhibiting TLR4 , NO and proinflammatory cytokine production than the nonpolar (hexane and diethyl ether) extract [62]. For the in vivo acute anti-inflammatory properties of $C$. nutans, the most effective dose was pretreatment of $9 \mathrm{mg} \mathrm{EPP} / \mathrm{ear}$ of methanol extract on the rat which resulted in $79 \%$ oedema inhibition at $15 \mathrm{~min}$ and $44.4 \%$ myeloperoxidase (MPO) reduction after $120 \mathrm{~min}$ of induction [70].

Additionally, in a comparison of the methanol, ethanol, and $n$-butanol extracts, a $1 \mathrm{~h}$ pretreatment of $200 \mathrm{mg} / \mathrm{kg}$ methanol extract administered via p.o. to carrageenaninduced rats was found to inhibit 59\% oedema formation [70]. The acetic acid-induced vascular permeability model showed that, among the water, methanol, chloroform, and $n$-butanol extracts, the butanol extract at $540 \mathrm{mg} / \mathrm{kg}$ was superior at exhibiting an anti-inflammatory effect $[68,69]$. Ethanol, aqueous ethanol, $n$-butanol, and cold creams have been evaluated for their subchronic anti-inflammation properties. The results showed that application of $125 \mathrm{mg}$ of C. nutans cold cream to a rat topically throughout the experimental period inhibited granuloma formation by as much as $50.98 \%$, which is comparable to the inhibitory effect of $0.25 \%$ prednisolone (56.82\%) [71]. According to the previous C. nutans anti-inflammatory studies, the extract prepared from polar solvent notably exhibited a promising anti-inflammatory properties in vitro (at a dose less than $30 \mu \mathrm{g} / \mathrm{mL}$ ) and in vivo (at a dose less than $300 \mathrm{mg} / \mathrm{kg}$ ). Therefore, it is greatly desired to focus on the relationship between the mode of action of $C$. nutans polar extract in both biological and nonbiological anti-inflammatory systems. In addition, since polar extract exhibited potential antiinflammatory activity, knowledge of extract prepared from the inorganic polar solvent particularly water is still scarce as regards its in vivo and in vitro anti-inflammatory activity properties, thus possessing potential research interest.

3.1.4. Immunomodulating Activity. Table 7(d) shows the immunomodulating effect of $C$. nutans. The methanol extract of $C$. nutans has been tested for its immune modulating effect on apoptosis and cytokines expression in experiments on human neutrophils and porcine peripheral blood mononuclear cells (PPBMCs), such as assays for fMLP macrophage activator-induced chemotaxis and chemokinesis, apoptosis, and concanavalin (ConA) and LPS-induced interleukin 10 (IL-10), and tumor necrosis factor-alpha (TNF- $\alpha$ ) expression [70, 72]. Wanikiat et al. [70] suggested that C. nutans methanol extract exhibited dose-dependent suppression of fMLP-induced chemotaxis and chemokinesis of neutrophils without causing the cells to undergo apoptosis. C. nutans was found to cause a reduction in IL-10 expression and to have no effect on TNF- $\alpha$ expression in PBMC [72]. On the other hand, the ethanolic extract of $C$. nutans has been tested for its immunomodulating effect on cytokines expression in splenocytes, a human keratinocyte cell line (HaCaT), and human peripheral blood mononuclear cells (HPBMC). Tu et al. [52] suggested that low concentrations of the ethanol extract cause IFN- $\gamma$ upregulation while higher concentration of $C$. nutans cause IFN- $\gamma$ downregulation. Sriwanthana et al. [73] studied found that low concentrations of C. nutans resulted in increase in lymphocyte proliferation, while higher concentrations of C. nutans resulted in decrease of lymphocyte production. The study also found that $C$. nutans did not stimulate an interleukin-2 (IL-2) response or affect the lymphocyte subpopulation, such as total T lymphocytes (CD3), $\mathrm{T}$ helper/inducer cells (CD4), T suppressor/cytotoxic cells (CD8), natural killer (NK) cells (CD16/CD56), or B lymphocytes (CD19). On the other hand, 2.5 and $5 \mathrm{mg} / \mathrm{mL}$ of ethanol extract increased the interleukin-4 (IL-4) production and 1 and $5 \mathrm{mg} / \mathrm{mL}$ of extract suppressed NK activity [73]. Furthermore, 1 and $100 \mu \mathrm{g} / \mathrm{mL}$ C. nutans were found to inhibit IFN- $\gamma$ and TNF- $\alpha$-induced keratinocytes apoptosis [63]. A similar trend as in the analgesic and anti-inflammatory properties of C. nutans could be observed, where extract prepared from polar solvents exerted better analgesic, anti-inflammatory, and immunomodulating effects. Phenolics, sulfur-containing glucosides, and sulfur-containing compounds might be the main contributors to these activities, as all the mentioned compounds were primarily isolated from polar C. nutans extracts. In addition, the described therapeutic effects are 
always well correlated with the immunology results. Thus, investigations on the biological mechanism of the active constituents present in the polar extract and their effects on immunological cells, mediators, and substances deserve special attention. Exploring the immunological function of other polar extracts, such as water, and nonpolar extracts should be encouraged as well.

3.1.5. Neuromodulating Activity. The efficacy of C. nutans in neuro-related protective and modulating functions has also been studied in recent years, as shown in Table 7(e). In vitro studies suggested that the neuroprotective effect of C. nutans was promising. From the studies by Tan et al. [74] and Tsai et al. [75], the $80 \%$ ethanol extract with a dose concentration less than $10 \mu \mathrm{g} / \mathrm{mL}$ was able to suppress post-hypoxic histone deacetylase (HDAC) activation and hypoxic neuronal death in an oxygen-glucose deprivation(OGD-) reoxygenation assay. It also decreased the levels of cytosolic phospholipase 2 (cPLA2) mRNA expression in mouse primary cortical neurons subjected to $0.5 \mathrm{~h}$ of OGD injury. In addition, Tan et al. [74] found that $100 \mu \mathrm{g} / \mathrm{mL}$ $80 \%$ ethanol extract was able to suppress histone acetylase (HAT) activity and regulate cPLA2 expression induction in a human neuroblastoma cell line (SH-SY5Y cells) through HDAC inhibitors such as entinostat (MS-275), MC-1568, and trichostatin A (TSA). The research team of Wu et al. [76] further suggested that treating the primary neurons with $0.15-20 \mu \mathrm{g} / \mathrm{mL}$ of $80 \%$ ethanol extract $1 \mathrm{~h}$ before, at the onset, or after the OGD induced cell death or OGD-reoxygenation treatment was able to mitigate the neuronal apoptosis and protected primary neurons by activating the antiapoptotic activity of peroxisome proliferator-activated receptor-gamma $($ PPAR- $\gamma) \rightarrow 14-3-3 \varepsilon$ (antiapoptotic marker), enhancing the $\mathrm{C} / \mathrm{EBP} \beta$ binding to PPAR- $\gamma$ promoter and amplifying its transcription though the extract was less effective when applied after the OGD treatment. The result also suggested that the protective effect of extract was revoked when cotreated with GW9662 (PPAR- $\gamma$ antagonist). This in vitro screening further complemented with the protective effect of extract in attenuating the ischemic brain damage in in vivo middle cerebral artery occlusion (MCA) stroke model [76]. Both intracerebroventricular (i.c.v.) infusion (10-60 pg extract) and i.p. injection ( $24 \mathrm{mg} / \mathrm{kg}$ body wt extract) of extract demonstrated that the extract possessed the ability in mitigating apoptotic neuronal death, cerebral infarct volume, and behavioral deficiency in the rat MCA occlusion model. The detailed neuroprotective mechanism of $C$. nutans was similar to in vitro OGD study and was elaborated in Table 7(e). In addition, an in vivo study conducted by Lau et al. [77] revealed that the three different tested doses of methanol extract were able to stimulate acetylcholinesterase activity in the heart, liver, and kidney, but not the brain of mice without inducing any signs of toxicity in the mice. As a short summary, similar results have been reported as those regarding the analgesic, anti-inflammatory, and immunomodulating activities, where the polar extract of $C$. nutans possessed a great neuroprotective effect. Nevertheless, research on the neuroprotective strength of $C$. nutans is still new and therefore holds enormous potential for different research directions. Further investigation of the potential efficacy of polar C. nutans extract in in vivo neurologyinflammation related studies might lead to a breakthrough. Research should fill in gaps about the neuro-related function of nonpolar extract as well.

3.1.6. Antidiabetic and $\alpha$-Glucosidase Inhibitory Activity. Table $7(\mathrm{f})$ shows a list of studies conducted on the antidiabetic and $\alpha$-glucosidase inhibition effects of $C$. nutans. There have been a total of 4 in vitro studies assessing the hyperglycemia inhibitory activity of $C$. nutans through an $\alpha$ glucosidase inhibition assay. All the results showed that $C$. nutans had a very low inhibitory effect on the $\alpha$-glucosidase enzyme when the employed extracts were prepared using conventional preparation methods $[4,28,37,61]$. Although $\mathrm{IC}_{50}$ was not calculated by Alam et al. [28], a lower $\mathrm{IC}_{50}$ value is anticipated as the extract prepared through advanced supercritical extraction exhibited $95.79 \%$ of $\alpha$-glucoside inhibition when the stock was prepared at $5000 \mu \mathrm{g} / \mathrm{mL}$. Despite C. nutans not showing particularly pronounced inhibition against $\alpha$-glucosidase, 3 in vivo antidiabetic experiments suggested that the water extract, $80 \%$ methanol extract, and the insoluble ethyl acetate fraction from the $80 \%$ ethanol extract were able to attenuate the insulin resistance induced by a high fat and high cholesterol diet (HFHC) and serum glucose level $[25,39]$. In general, the high $\alpha$-glucosidase inhibition by SFE treated extracts might indicate that the compounds active against the $\alpha$-glucosidase enzyme are heat-sensitive or easily degradable. Thus, extra precaution is needed during the extract preparation step. On the other hand, conventional method prepared extracts that mimicked the traditional application showed low inhibition towards in vitro $\alpha$-glucosidase tests while traditional uses suggested that C. nutans is an antidote for diabetes. The contradictory results might be attributed by improper mode of action of diabetes being investigated. Since $\alpha$-glucosidase inhibition test focuses on mechanism of postprandial hyperglycemia, further study on other mechanism approach, experiment on the reaction of the extract towards $\beta$-cell in pancreas, test that involved other enzymes in hyperglycemia such as pancreatic $\alpha$-amylase, glucose diffusion assay, glucose uptake by yeast cells, and nonenzymatic glycosylation assay are highly recommended.

3.1.7. Antioxidant Activity. Considerable number of analyses have been done on the antioxidant effect of $C$. nutans as shown in Table $7(\mathrm{~g})$. Based on the 2,2-diphenyl-1picrylhydrazyl (DPPH) free radical assay, some studies showed that $C$. nutans up to $1 \mathrm{mg} / \mathrm{mL}$ is a potential antioxidant agent, while other studies found that the DPPH inhibitory properties of $C$. nutans could not be determined $[47,64,78]$. The data in the table showed that, among the polar and nonpolar extracts tested, the highest antioxidant properties of $C$. nutans was detected in the study by Ghasemzadeh et al. [41], where C. nutans bud extract that was cultivated for 1 year at a farm located in Malaysia and that underwent freeze drying and methanol extraction resulted in $\mathrm{IC}_{50}$ value of $64.6 \mu \mathrm{g} / \mathrm{mL}$. The second most common 
antioxidant determination assay selected by researchers was the ferric reducing antioxidant power (FRAP) assay. However, discussion of the FRAP results is difficult, as there is a lack of unit standardization in the measurement. The results from others in vitro antioxidant assays, such as those for hydrogen peroxide scavenging, metal chelating, nitric oxide scavenging, 2,2' -azino-bis(3-ethylbenzothiazoline-6sulfonic acid) (ABTS) cation radical scavenging, galvinoxyl radical scavenging activity, superoxide radical scavenging activity, and phorbol 12-myristate 13-acetate (PMA) induced peroxide production in rat macrophages and protective effect against peroxyl radicals initiator- (AAPH-) induced oxidative hemolysis, have been summarized in Table $7(\mathrm{~g}(3)-\mathrm{g}(10))$. Overall, polar and semipolar solvent-extracted $C$. nutans were more likely to exhibit moderate antioxidant activity at concentration varying from 0.0125 to $10 \mathrm{mg} / \mathrm{mL}$ than nonpolar extracts. In addition, there was a recent study that evaluated the antioxidant activity of $C$. nutans in vivo by using a hyperlipidemia-associated oxidative stress model [42]. The study suggested that both the water and $80 \%$ methanol leaf extracts at up to $500 \mathrm{mg} / \mathrm{kg}$ bw rats were able to mitigate oxidative stress by improving the activity of serum antioxidant enzymes and expression of hepatic antioxidant genes [42]. In summary, polar and semipolar extracts are more likely to exhibit antioxidant properties. A possible hypothesis is that phenolics, sulfur-containing glucosides, sulfur-containing compounds, chlorophyll derivatives, and some phytosterol derivatives that are isolated from C. nutans using polar and semipolar solvents might be the contributors. Thus, studies on the antioxidant properties of these isolated compounds and further evaluation of the plant's antioxidant effect in in vivo studies are required.

3.1.8. Antiviral Activity. The antimicrobial properties of $C$. nutans have been further classified into antiviral, antibacterial, and antifungal effects, as shown in Tables 7(h), 7(i), and $7(j)$, respectively. For the antiviral activity of $C$. nutans, the types of virus that have been studied were varicella zoster virus (VZV), herpes simplex virus (HSV), fish pathogenic viruses, crustaceans (shrimp and prawn) infectious viruses, mosquito-borne viruses, and poultry and bird contagious viruses. Notably, the antivirus studies were always accompanied with a prescreening test for cytotoxicity to determine the subtoxic concentration of the test sample and to ensure that the test sample has a killing effect on the virus instead of on the host cell [79]. Researchers have also investigated the mode of action of $C$. nutans in terms of its antiviral potential through 3 different stages of treatment. Pretreatment (pre-) studies are those where the test sample is allowed to incubate with the cell for a time of period before it is infected with the virus. This approach reflects the virucidal activity of the test sample, as the test sample interferes with the viral structure to prevent or inhibit the viral penetrating or adsorbing to the host cell [79]. In posttreatment (post-) studies, instead of being preincubated with the test sample, the virus is attached to the host cell that has first only been cultured with the test sample. The test sample is thus considered to have antiviral activity if it successfully inhibits viral DNA replication [79].
By contrast, for the direct inactivation (direct) pathway, the virus is preincubated with the test samples first before being added to the host cell. The test sample is said to have an inactivation ability against the virus if it interferes with or causes damage to the viral glycoproteins, the virus envelope, or the virus structure before they enter the host cell [79].

Thus far, only one in vitro study and a total of 3 clinical trials have been conducted to evaluate C. nutans' anti-VZV activity. The in vitro result suggested that the organic extract exhibited anti-VZV activity through the direct inactivation stage [80]. In the clinical studies, the C. nutans extracts were formulated into a $5 \%$ C. nutans cream prior to testing its ability to combat VZV infections. The result suggested that the $C$. nutans containing cream was able to exhibit a positive curing effect. All the studies showed that the percentage of patients who experienced lesion crusting within 3 days and lesion healing within 7 days after applying the C. nutans cream topically was better than the percentage in the placebo and acyclovir groups. In addition, the pain score and side effect resulting from $C$. nutans cream application were lower $[8,81,82]$.

For the anti-HSV-1 activity of C. nutans, the ethyl acetate, methanol, dichloromethane, chloroform, and $n$ hexane extracts have been evaluated. Thongchai et al. [79] suggested that the $C$. nutans ethyl acetate leaf extract displayed the best inhibition on HSV strain with $\mathrm{IC}_{50}$ value of $7.6 \mu \mathrm{g} / \mathrm{mL}$ through the pretreatment mechanism. Meanwhile, the methanol, ethanol dichloromethane, chloroform, and hexane extracts were also tested for their anti-HSV-2 activity. The $C$. nutans methanol and hexane leaf extracts were found to have better and comparable inhibition against HSV-2Baylor 186 strain, via posttreatment action, with $\mathrm{IC}_{50}$ value of 65.13 and $72.62 \mu \mathrm{g} / \mathrm{mL}$, respectively [83]. The anti-HSV2 activity of $C$. nutans has also been evaluated in clinical studies using the $5 \%$ extract cream. The results obtained were similar to those from the clinical studies on the anti-VZV activity; when compared to a placebo, the days required for lesion crusting and healing after application of C. nutans were higher than or the same in terms of efficacy as acyclovir [82, 84, 85]. Regarding its efficacy in inhibiting fish pathogenic viruses, among the virus strains tested, the $C$. nutans ethanolic extract exerted $100 \%$ inhibition on plaque formation in infectious hematopoietic necrosis virus (IHNV) and Oncorhynchus masou virus (OMV) strains but not in infectious pancreatic necrosis virus (IPNV) through the direct inactivation stage.

On the other hand, it was also found that C. nutans ethanol extract had an excellent protective effect on cultured black tiger shrimp against yellow head rhabdo-like virus (YRV-RNA) through a direct inactivation mechanism and pronounced virucidal activity against dengue virus $\left(\mathrm{IC}_{50}\right.$ value of $31.04 \mu \mathrm{g} / \mathrm{mL}$ ); however, it was less effective against Newcastle disease virus (NDV) caused by poultry and birds [52, 86, 87]. In summary, C. nutans ethanol extract possessed a very promising antiviral effect. Future research may explicate the antiviral activity of stigmasterol derivative, sulfur-containing compounds, monoacylmonogalactosylgycerol, and all of nine cerebrosides, as, according to previous studies, they are the main compounds isolated 
from the ethanol extract. Though clinical trial suggested its therapeutic properties, research that optimizes the efficacy dosage and profiles the safety uses of the plant extract is lacking.

3.1.9. Anti-Bacterial Activity. Table 7(i) shows the antibacterial properties of $C$. nutans. Overall, studies were mainly based on in vitro assays and only polar (water, methanol, and ethanol) and semipolar (chloroform and ethyl acetate) extracts, but nonpolar C. nutans extracts not were evaluated for their anti-bacterial activity. A total of 9 different Gram-positive bacteria have been used in previous studies, including Bacillus cereus, Bacillus subtilis, Staphylococcus aureus, methicillin-resistant Staphylococcus aureus (MRSA), methicillin-sensitive Staphylococcus aureus (MSSA), Micrococcus luteus, Propionibacterium acnes, Staphylococcus epidermidis, and Streptococcus sp. On the other hand, Aeromonas hydrophila, Escherichia coli and its strains, Neisseria gonorrhoeae, Pseudomonas aeruginosa and its strains, Salmonella enterica and its serovars, and Vibrio harveyi and Vibrio parahaemolyticus were the Gram-positive bacteria selected for study. As a summary of the antibacterial efficacy of $C$. nutans, the most effective extract of the plant regarding antibacterial effect was prepared by oven-drying and undergoing ethyl acetate fractionation. The extract prevented visible $B$. cereus, E. coli, and S. enterica Typhimurium growth at $1.39 \mathrm{mg} / \mathrm{mL}$ [88].

3.1.10. Antifungal Activity. Concerning the antifungal efficacy of $C$. nutans, limited studies had been carried out to date. In the two reported antifungal activity studies, Cheeptham and Towers [89] found that C. nutans did not exert a fungicidal effect on Candida albicans or Aspergillus fumigatus when $5 \mathrm{mg} / \mathrm{mL} \mathrm{95 \%} \mathrm{ethanol} \mathrm{leaf} \mathrm{extract} \mathrm{was} \mathrm{tested.} \mathrm{By} \mathrm{contrast,} \mathrm{a}$ fraction of the ethyl acetate extract at the minimal concentration of $1.39 \mathrm{mg} / \mathrm{mL}$ did exhibit an antifungal effect on $C$. albicans [88]. In summary, based on the available in vitro studies, C. nutans polar and semipolar extracts exhibited a weak antibacterial and antifungal activities. Any further research should focus on assessing the biological action of the polar and semipolar extracts of $C$. nutans in inhibiting bacterial and fungal infections as well as augmenting the limited information on the antibacterial and antifungal potency of the nonpolar extract.

3.1.11. Anticancer Activity. A total of 15 types of cancer cells that are responsible for different types of cancer have been used to evaluate the in vitro anticancer effect of $C$. nutans, as summarized in Table $7(\mathrm{k})$. These include human melanoma cell lines (D24 and MM418C1) for skin cancer, a human osteosarcoma cell line (cultured Saos-2) for bone tumor, human breast adenocarcinoma (MCF7) and human breast carcinoma (BT474) cell line for breast cancer, human lung adenocarcinoma (NCI-H23) and human lung carcinoma (NCI-H460) cell lines for lung cancer, a human liver hepatocellular carcinoma cell line (HepG2) for liver cancer, a human neuroblastoma cell line (IMR-32) for nerve tissue cancer, a human gastric cancer cell line (SNU-1) for gastric cancer, human colon adenocarcinoma (LS-174T) and human colorectal carcinoma cell line (HCT 116) for colon and rectal cancer, a human cervical cancer cell line (HeLa) for cervical cancer, a human erythroleukemia cell line (K562) for acute myeloid leukemia, and a human Burkitt's lymphoma cell line (Raji) for lymphatic disorder. In addition, there were 2 studies that assessed the in vivo antimutagenic and anticancer effect of $C$. nutans $[33,90]$. Based on these studies, the most effective extract was the petroleum ether leaf extract, which showed the strongest cytotoxic activity after $72 \mathrm{~h}$ of incubation. The concentration of the extract that caused $50 \%$ death $\left(\mathrm{CC}_{50}\right)$ was $18.0 \mu \mathrm{g} / \mathrm{mL}$ in HeLa cell and $20.0 \mu \mathrm{g} / \mathrm{mL}$ in the $\mathrm{K}-562$ cell line [88]. According to the National Cancer Institute (NCI), a crude extract that exhibits an $\mathrm{IC}_{50}$ value $<20 \mu \mathrm{g} / \mathrm{mL}$ can be considered as an active anticancer agent [41]. Overall, nonpolar leaf extract possessed a very pronounced cytotoxic effect on cervical and erythroleukemia cancerous cell. Hence, to evaluate the suitability of $C$. nutans to involve in anticancer drug development, further in vivo clinical experimental studies on cervical cancer and erythroleukemia models are encouraged. The mechanism behinds the activity should be critically analyzed too. On the other hand, from the summarized data, observed inconsistency of incubation period during the experiment might be one of the factors that caused result deviation. Further parameter standardization should be implemented. Furthermore, the effect of the ethanol extract on in vivo hepatocarcinoma tumor-bearing mice exhibited a similar trend as in the in vitro study (methanolic extract) suggesting that both polar extracts possessed similar cytotoxic action towards liver cancerous cells, which is worthy of further investigation.

3.1.12. Wound Healing Activity. In addition to the other well studied pharmacological activities of C. nutans, there are also a few other potential pharmacological activities which are less studied or that even have been reported only once. Table 7(l) summarizes the wound healing ability of C. nutans. Between the chloroform extract and hexane extract, the chloroform extract $(10 \mu \mathrm{g} / \mathrm{mL})$ was found to provide the best improvement in human gingival fibroblast (HGF) migration rate and wound recovery in the $6 \mathrm{~h}$ of observation [11]. Before labelling it as a potential wound healing agent during inflammation, however, more studies are needed to verify its wound healing effect and determine which of the chlorophyll derivatives, diglycerides, or stigmasterol derivatives are the main factor for this positive activity.

3.1.13. Protective Effect on Plasmid DNA Activity. The protective effect of $C$. nutans on plasmid DNA is tabulated in Table $7(\mathrm{~m})$. Yuann et al. [64] suggested that, compared to green tea extract (protection up to $30 \mathrm{~min}$ ), the $70 \%$ ethanol extract had the ability to reduce the number of DNA cleavages, preserve higher levels of supercoiled plasmid DNA integrity, and provide better protection against the riboflavin photoreaction induced superoxide for up to $50 \mathrm{~min}$. In short, the polar extract showed a protective effect on plasmid DNA, but more analysis involving an in vivo model is needed. In 
addition, it is important to direct research focus towards the protective effect of the nonpolar extract as well.

3.1.14. Lipid Elevated Inhibition Activity. The ability of $C$. nutans to attenuate obesity had been assessed as shown in Table 7(n) through an in vitro porcine pancreatic lipase inhibition assay. The result suggested that, instead of an inhibitory effect, the methanol extract exhibited a pancreatic lipase promoting effect [21]. However, as suggested previously, more research involving cellular and in vivo experimental design are encouraged before reaching any conclusion.

3.1.15. Oral Mucositis and Stomatitis Treatment. C. nutans is also formulated for oral application to treat radiation induced oral mucositis in head and neck cancer patients and in patients suffering from recurrent aphthous stomatitis, as presented in Table $7(0)[10,91]$. The results revealed that, compared to the positive control group, the time onset of oral mucositis was significantly later, and the pain score was significantly lower [10]. Although it is not as efficacious as the positive control group (triamcinolone acetonide), it can be a good alternative to lessen the severity of ulcers [91].

\subsection{Pharmacological Activity of C. nutans Isolated Pure Com-} pounds. After understanding the pharmacological properties of $C$. nutans extract, it is beneficial to further investigate which compound in a particular extract is responsible for the therapeutic activity of the plant. Table 8 summarizes the pharmacological activities of the isolated compounds from C. nutans. For anti-inflammatory activity, only cerebrosides from the ethanol extract have been studied, and they were reported as being ineffective in suppressing cyclooxygenase (COX-1 and COX-2) induced prostaglandin E2 generation and HSV-1 infection [55]. The result obtained was inconsistent with the anti-inflammatory effect of the extract observed previously. Nevertheless, the type of experiment conducted might cause this variation, and further attention should focused on the anti-inflammatory effect of other compounds isolated from the polar solvent. Le et al. [45] studied the immunomodulating function of flavones (schaftoside) and terpenes (stigmasterol, $\beta$-sitosterol, and lupeol) compounds isolated from the hexane fraction of the methanol extract. The results showed that only stigmasterol and $\beta$-sitosterol were able to suppress the $\mathrm{T}$ cell proliferation mediated by ConA and that only $\beta$-sitosterol exerted an immune suppressive effect on T helper 2 (Th2) cytokines (IL-4 and IL-10). A similar trend was observed with the polar extract of $C$. nutans which exerted a better immunomodulating effect than the nonpolar extract. Clinamide $\mathrm{D}$, which was isolated from the methanol extract, was found to have a moderate antioxidant effect with $76.05 \pm 0.02 \% \mathrm{DPPH}$ radical inhibition at $1000 \mu \mathrm{g} / \mathrm{mL}$ which is consistent with the good DPPH inhibition properties of the methanol extract $[41,53]$.

Regarding the antiviral activity, $13^{2}$-hydroxy- $\left(13^{2}-\mathrm{R}\right)$ phaeophytin $b$ from the chloroform extract was found to possess a better anti-HSV-1 effect with an $\mathrm{IC}_{50}$ value of $1.96 \mathrm{nM}$ through direct inactivation [107]. Pongmuangmul et al. [56] showed that monogalactosyl diglyceride (MGDG) and digalactosyl diglyceride (DGDG) isolated from the chloroform extract exhibited promising anti HSV-1 and antiHSV-2 properties in the poststep of infection, where the $\mathrm{IC}_{50}$ values were in a range from $36.00 \mu \mathrm{g} / \mathrm{mL}$ to $43.20 \mu \mathrm{g} / \mathrm{mL}$. Sittiso et al. [108] suggested that phaeophorbide A from the chloroform extract was able to inhibit dengue viral 2 replication in the direct inactivation and postincubation stages with a $\mathrm{CC}_{50}$ of $25 \mu \mathrm{g} / \mathrm{mL}$. Based on this study, it can be observed that, in antiviral studies, compounds isolated from the chloroform extract are more likely to exhibit antiviral activity whereas the ethyl acetate, methanol, and ethanol extracts were more likely to possess anti-HSV-1 and antiHSV-2 activities, and the ethanol extract was more likely to possess an anti-dengue inhibitory effect. Thus, it might be a valuable approach to investigate the relationship between that antiviral properties of compounds isolated from the aforementioned extracts. Tinh [49] found that 3-amino-4,5dihydroxyfuran-2 $(3 \mathrm{H})$-one isolated from the ethanol extract exhibited moderate antibacterial activity against $S$. aureus (MIC equal to $0.3125 \mathrm{mg} / \mathrm{mL}$ ). This result was inconsistent with the antibacterial activity of the corresponding extract against the same bacterial strain. This might due to the antibacterial properties of the crude extract being hindered by the other constituents present in the extract. In addition, phaeophorbide A from the chloroform extract was found to possess a promising in vitro anticancer effect on human lung carcinoma cell (A549 cells) at a CC 50 equal to $25 \mu \mathrm{g} / \mathrm{mL}$ [108]. This result was consistent with the study from Yong et al. [32] which found that the chloroform extract at $100 \mu \mathrm{g} / \mathrm{mL}$ showed $55.82 \%$ inhibition of human lung cancer cell. A novel polysaccharide-peptide complex (CNP-1-2) isolated by Huang et al. [59] from the $70 \%$ ethanol extract also exhibited potential anticancer activity on a human gastric cancer cell line (SGC-7901 cells), where, at concentration of $200 \mu \mathrm{g} / \mathrm{mL}$, it inhibited cell growth by $92.34 \pm 0.94 \%$ after $48 \mathrm{~h}$ of incubation. By contrast, Yong et al. [32] found that the chloroform extract exhibited a weak killing effect on human gastric cancer cells $(31.25 \pm 1.09 \%$ at $100 \mu \mathrm{g} / \mathrm{mL})$.

\section{Conclusion}

Phytochemical identification and quantification have suggested that C. nutans is a rich source of phenolics, flavonoids, triterpenoids, and chlorophyll derivatives; however, their retention in an extract is largely influenced by the plant preparation methods. On the other hand, pharmacological studies on C. nutans have suggested that its polar extracts can be a promising anti-inflammation, antiviral, anticancer, immune- and neuromodulating, and plasmid DNA protective agents, as well as a moderate antivenom, analgesic, antidiabetic, and antioxidant agent and a weak lipid elevating inhibitor. By contrast, its semipolar extracts can be a promising antiviral, anticancer and wound healing agent, a moderate anti-inflammation and antioxidant agent, and a weak antibacterial and antifungal agent, and its nonpolar extracts can be a strong anticancer agent. However, as insufficient previous scientific studies have been conducted and most experiments were preliminary and fundamentally oriented, 
more sophisticated evaluation and pathway analyses of the aforementioned biological and therapeutic potential of this plant are urged before implementing it in the pharmaceutical and cosmetics industries. In addition, since in vitro assays do not fully mimic the physiological environment in animals and humans, additional cellular, in vivo and clinical trials are likewise to fully interpret the effect of $C$. nutans on disease inhibition and prevention. Mechanism of action of the extract towards particular treatment should explicate. It is also likely to have more experimental studies that could substantiate and describe the correlation of the isolated phytochemicals from C. nutans with their corresponding pharmacological effects. A note for future researchers: it is of utmost importance to provide complete data such as the extract concentration, the extraction solvent used, and the experimental design, which was found to be lacking throughout the data searching. All the aforementioned data could be a crucial point that determines the accuracy of the interpretation of the results, the credibility of the study, and the reproducibility of the work in the future. In conclusion, through this review, the authors hope to provide a more systematic summary of the previous C. nutans works according to the experimental design and plant preparation methods that have been thus far done in phytochemical and pharmacological relevant C. nutans studies. The authors also anticipate to provide some possible idea for researchers regarding the future research perception and direction of this plant.

\section{Conflicts of Interest}

The authors declared no conflicts of interest.

\section{Acknowledgments}

The first author gratefully acknowledges support by the Ministry of Education (MOE), Malaysia, for the scholarship provided under Mybrain-MyPhD scheme. This work was supported by Ministry of Agriculture (MOA), Malaysia [NH1014D067, January 2015-June 2017], under the NRGS scheme.

\section{References}

[1] S. Sharma, Plant Taxonomy, Pacific Book International, New Delhi, India, 2nd edition, 2011.

[2] A. Alam, S. Ferdosh, K. Ghafoor et al., "Clinacanthus nutans: A review of the medicinal uses, pharmacology and phytochemistry," Asian Pacific Journal of Tropical Medicine, vol. 9, no. 4, pp. 402-409, 2016.

[3] L. C. Green, D. A. Wagner, J. Glogowski, P. L. Skipper, J. S. Wishnok, and S. R. Tannenbaum, "Analysis of nitrate, nitrite, and $\left[{ }^{15} \mathrm{~N}\right]$ nitrate in biological fluids," Analytical Biochemistry, vol. 126, no. 1, pp. 131-138, 1982.

[4] L. W. Khoo, A. Mediani, N. K. Z. Zolkeflee et al., "Phytochemical diversity of Clinacanthus nutans extracts and their bioactivity correlations elucidated by NMR based metabolomics," Phytochemistry Letters, vol. 14, pp. 123-133, 2015.

[5] C. Cherdchu, N. Poopyruchpong, R. Adchariyasucha, and K. Ratanabanangkoon, "The absence of antagonism between extracts of Clinacanthus nutans Burm. and Naja naja siamensis venom," Southeast Asian Journal of Tropical Medicine and Public Health, vol. 8, no. 2, pp. 249-254, 1977.

[6] S. Y. Ho, W. P. Tiew, P. Madhavan, M. S. Abdul Shukkoor, and G. A. Akowuah, "Phytochemical analysis and antibacterial activity of methanolic extract of Clinacanthus nutans leaf," International Journal of Drug Development and Research, vol. 5, no. 3, pp. 349355, 2013.

[7] H. A. Levey, "Toxicity of the venom of the sea-snake, Laticauda colubrina, with observations on a Malay'folk cure," Toxicon, vol. 6, no. 4, pp. 269-276, 1969.

[8] S. Sangkitporn, S. Chaiwat, K. Balachandra, T. D. Na-Ayudhaya, M. Bunjob, and C. Jayavasu, "Treatment of herpes zoster with Clinacanthus nutans (bi phaya yaw) extract," Journal of the Medical Association of Thailand, vol. 78, no. 11, pp. 624-627, 1995.

[9] K. Panyakom, Structural Elucidation of Bioactive Compounds of Clinacanthus nutans (Burm.F.) Lindau Leaves, Suranaree University of Technology, 2006.

[10] P. Putwatana, P. Sanmanowong, O. Ladawal, J. Tiraporn, P. Supaporn, and N. Ladawan, "Relief of radiation-induced oral mucositis in head and neck cancer," Cancer Nursing, vol. 32, no. 1, pp. 82-87, 2009.

[11] M. O. Roeslan, T. D. Na Ayudhya, and K. Sittichai, "Characteristics of Clinacanthus nutans extraction from Thailand and Indonesia (preliminary study)," in Proceedings of the Conference on Graduate Student Network of Thailand, 2012.

[12] S. Y. Shim, I. Aziana, and B. Y. Khoo, "Perspective and insight on Clinacanthus nutans Lindau in traditional medicine," International Journal of Integrative Biology, vol. 14, no. 1, pp. 7-9, 2013.

[13] M. S. Aslam, M. S. Ahmad, and A. S. Mamat, "A review on phytochemical constituents and pharmacological activities of Clinacanthus nutans," International Journal of Pharmacy and Pharmaceutical Sciences, vol. 7, no. 2, pp. 30-33, 2015.

[14] R. Yahaya, G. K. Dash, M. S. Abdullah, and A. Mathews, "Clinacanthus nutans (burm. F.) Lindau: An useful medicinal plant of south-east Asia," International Journal of Pharmacognosy and Phytochemical Research, vol. 7, no. 6, pp. 1244-1250, 2015.

[15] E. Farsi, A. S. Abdul Majid, and A. M. S. Abdul Majid, "Clinacanthus nutans, yesterday's practice, and future's drug: A comprehensive review," American Journal of Phytomedicine and Clinical Therapeutics, vol. 4, no. 4, pp. 113-126, 2016.

[16] I. N. Zulkipli, R. Rajabalaya, A. Idris, N. A. Sulaiman, and S. R. David, "Clinacanthus nutans: A review on ethnomedicinal uses, chemical constituents and pharmacological properties," Pharmaceutical Biology, vol. 55, no. 1, pp. 1093-1113, 2017.

[17] A. Shuyporm, Chemical Composition Investigation of the Clinacanthus nutans (Burm. F.) Lindau Leaves, Suranaree University of Technology, 2004.

[18] S. Y. Fong, Genetic, Phytochemical and Bioactivity Studies of Clinacanthus nutans (Burm. F.) Lindau (Acanthaceae), RMIT University, 2015.

[19] M. H. Abdul Rahim, Z. A. Zakaria, M. H. Mohd Sani et al., "Methanolic extract of Clinacanthus nutans exerts antinociceptive activity via the opioid/nitric oxide-mediated, but cGMPindependent, pathways," Evidence-Based Complementary and Alternative Medicine, vol. 2016, Article ID 1494981, pp. 1-11, 2016.

[20] N. Keawpradub and J. Purintrapiban, "Upregulation of glucose uptake in L8 myotubes by the extract from lagerstroemia 
speciosa: A possible mechanism of action," Maejo International Journal of Science and Technology, vol. 3, no. 3, pp. 472-485, 2009.

[21] S. L. Ong, S. Paneerchelvan, H. Y. Lai, and N. K. Rao, "In vitro lipase inhibitory effect of thirty two selected plants in Malaysia," Asian Journal of Pharmaceutical and Clinical Research, vol. 7, no. 2, pp. 19-24, 2014.

[22] M. Sekar and N. A. Rashid, "Formulation, evaluation and antibacterial properties of herbal ointment containing methanolic extract of Clinacanthus nutans leaves," International Journal of Pharmaceutical and Clinical Research, vol. 8, no. 8, pp. 1170-1174, 2016.

[23] J. L. Chelyn, M. H. Omar, N. S. A. Mohd Yousof, R. Ranggasamy, M. I. Wasiman, and Z. Ismail, "Analysis of flavone $C$-glycosides in the leaves of Clinacanthus nutans (Burm. f.) Lindau by HPTLC and HPLC-UV/DAD," The Scientific World Journal, vol. 2014, Article ID 724267, pp. 1-6, 2014.

[24] W. P. Tiew, P. X. Wen, J. H. Chin, and G. A. Akowuah, "Effect of methanol extract of Clinacanthus nutans on serum biochemical parameters in rats," Journal of Applied Pharmacy, vol. 6, no. 1, pp. 77-86, 2014.

[25] Y. Nurulita, H. Dhanutirto, and A. A. Soemardji, "Refining activity and antidiabetic compound leaf aqueous extract Dandang Gendis," Jurnal Natur Indonesia, vol. 10, no. 2, pp. 98-103, 2008.

[26] S. Goonasakaran, Preliminary Antimicrobial And Phytochemical Analysis of Clinacanthus nutans and Azadirachta indica, Universiti Teknologi Malaysia, 2013.

[27] P. Dampawan, C. Huntrakul, and V. Reutrakul, "Constituents of Clinacanthus nutans and the crystal structure of lup-20(29)ene-3-one," Journal of the Science Society of Thailand, vol. 3, no. 1, pp. 14-26, 1977.

[28] M. A. Alam, I. S. M. Zaidul, K. Ghafoor et al., "Identification of bioactive compounds with GC-Q-TOF-MS in the extracts from Clinacanthus nutans using subcritical carbon dioxide extraction," Separation Science and Technology (Philadelphia), vol. 52, no. 5, pp. 852-863, 2017.

[29] B. E. Cheong, S. Y. Ho, and V. F. Dickens Wong, "Chemical profiling of Sabah snake grass, Clinacanthus nutans," in Proceedings of the 11th Seminar on Science \& Technology, 2013.

[30] A. N. Mustapa, Á. Martin, R. B. Mato, and M. J. Cocero, "Extraction of phytocompounds from the medicinal plant Clinacanthus nutans Lindau by microwave-assisted extraction and supercritical carbon dioxide extraction," Industrial Crops and Products, vol. 74, pp. 83-94, 2015.

[31] P. L. Teoh, A. Y. F. Cheng, M. Liau et al., "Chemical composition and cytotoxic properties of Clinacanthus nutans root extracts," Pharmaceutical Biology, vol. 55, no. 1, pp. 394-401, 2017.

[32] Y. K. Yong, J. J. Tan, S. S. Teh et al., "Clinacanthus nutans extracts are antioxidant with antiproliferative effect on cultured human cancer cell lines," Evidence-Based Complementary and Alternative Medicine, vol. 2013, pp. 1-8, 2013.

[33] D. Huang, W. Guo, J. Gao, J. Chen, and J. Olatunji, "Clinacanthus nutans (Burm. f.) Lindau ethanol extract inhibits hepatoma in mice through upregulation of the immune response," Molecules, vol. 20, no. 9, pp. 17405-17428, 2015.

[34] E. A. Ainsworth and K. M. Gillespie, "Estimation of total phenolic content and other oxidation substrates in plant tissues using Folin-Ciocalteu reagent," Nature Protocols, vol. 2, no. 4, pp. 875-877, 2007.
[35] H. A. Hamid and I. H. Yahaya, "Cytotoxicity of Clinacanthus nutans extracts on human hepatoma (HepG2) cell line," International Journal of Pharmacy and Pharmaceutical Sciences, vol. 8, no. 10, pp. 8-10, 2016.

[36] M. Lusia Barek, M. Hasmadi, A. Z. Zaleha, and A. B. Mohd Fadzelly, "Effect of different drying methods on phytochemicals and antioxidant properties of unfermented and fermented teas from Sabah snake grass (Clinacanthus nutans Lind.) leaves," International Food Research Journal, vol. 22, no. 2, pp. 661-670, 2015.

[37] F. C. Wong, A. L. Yong, E. P. S. Ting et al., "Antioxidant, metal chelating, anti-glucosidase activities and phytochemical analysis of selected tropical medicinal plants," Iranian Journal of Pharmaceutical Research, vol. 13, no. 4, pp. 1407-1413, 2014.

[38] K. B. Raya, S. H. Ahmad, S. F. Farhana, M. Mohammad, N. E. Tajidin, and A. Parvez, "Changes in phytochemical contents in different parts of Clinacanthus nutans (Burm. f.) lindau due to storage duration," Bragantia, vol. 74, no. 4, pp. 445-452, 2015.

[39] N. Sarega, M. U. Imam, N. M. Esa, N. Zawawi, and M. Ismail, "Effects of phenolic-rich extracts of Clinacanthus nutans on high fat and high cholesterol diet-induced insulin resistance," BMC Complementary and Alternative Medicine, vol. 16, no. 1, 88 pages, 2016.

[40] Q. Yu, Z. Duan, W. Duan, F. Shang, and G. Yang, "Analysis and evaluation of nutrition composition of Clinacanthus nutans," in Proceedings of the 2nd Technical Congress on Resources, Environment and Engineering, CREE 2016, pp. 369-373, 2016.

[41] A. Ghasemzadeh, A. Nasiri, H. Z. E. Jaafar, A. Baghdadi, and I. Ahmad, "Changes in phytochemical synthesis, chalcone synthase activity and pharmaceutical qualities of sabah snake grass (Clinacanthus nutans L.) in relation to plant age," Molecules, vol. 19, no. 11, pp. 17632-17648, 2014.

[42] N. Sarega, M. U. Imam, D.-J. Ooi et al., "Phenolic rich extract from Clinacanthus nutans attenuates hyperlipidemiaassociated oxidative stress in rats," Oxidative Medicine and Cellular Longevity, vol. 2016, Article ID 4137908, 2016.

[43] F.-C. Wong, A.-L. Yong, H.-C. Ong, and T.-T. Chai, "Evaluation of the antibacterial activities of selected medicinal plants and determination of their phenolic constituents," ScienceAsia, vol. 39, no. 6, pp. 591-595, 2013.

[44] G. Boonkerd, The Chemical Constituents of Clinacanthus burmanii, Chulalongkorn University, 1967.

[45] C.-F. Le, T. H. Kailaivasan, S.-C. Chow, Z. Abdullah, S.-K. Ling, and C.-M. Fang, "Phytosterols isolated from Clinacanthus nutans induce immunosuppressive activity in murine cells," International Immunopharmacology, vol. 44, pp. 203-210, 2017.

[46] J. Lin, H. M. Li, and J. G. Yu, "Studies on the chemical constituents of niu xu hua (Clinacanthus nutans)," Zhongcaoyao, vol. 14, pp. 337-338, 1983.

[47] P. N. M. Dan, Evaluation of Biological Activities of Clinacanthus nutans (Burm.F.) Lindau, Vietnam National University, 2014.

[48] P. Dampawan, Studies of the Chemical Constituents of Clinacanthus nutans (acanthaceae) and Zingiber cassumunar Roxb. (zingiberaceae), Mahidol University, 1976.

[49] T. D. D. Tinh, Biological Activities of Clinacanthus nutans (Burm. F) Lindau Extracts, Vietnam National University in HCMC, 2014.

[50] K. Teshima, T. Kaneko, K. Ohtani et al., "C-glycosyl flavones from Clinacanthus nutans," Natural Medicine Note, vol. 51, no. 6, article 557, 1997. 
[51] K.-I. Teshima, T. Kaneko, K. Ohtani et al., "Sulfur-containing glucosides from Clinacanthus nutans," Phytochemistry, vol. 48, no. 5, pp. 831-835, 1998.

[52] S.-F. Tu, R. H. Liu, Y.-B. Cheng et al., "Chemical constituents and bioactivities of Clinacanthus nutans aerial parts," Molecules, vol. 19, no. 12, pp. 20382-20390, 2014.

[53] H. A. Hamid, I. H. Yahya, M. M. Yusoff, and S. Zareen, "Bioassay-guided isolation and antioxidant activity of sulfurcontaining compounds from Clinacanthus nutans," Journal of the Chinese Chemical Society, vol. 63, no. 12, pp. 1033-1037, 2016.

[54] S. Satakhun, Chemical Constituents of Clinacanthus nutans Leaves, Chulalongkorn University, 2001.

[55] P. Tuntiwachwuttikul, Y. Pootaeng-On, P. Phansa, and W. C. Taylor, "Cerebrosides and a monoacylmonogalactosylglycerol from Clinacanthus nutans," Chemical \& Pharmaceutical Bulletin, vol. 52, no. 1, pp. 27-32, 2004.

[56] S. Pongmuangmul, S. Phumiamorn, P. Sanguansermsri, N. Wongkattiya, I. H. Fraser, and D. Sanguansermsri, "Antiherpes simplex virus activities of monogalactosyl diglyceride and digalactosyl diglyceride from Clinacanthus nutans, a traditional Thai herbal medicine," Asian Pacific Journal of Tropical Biomedicine, vol. 6, no. 3, pp. 192-197, 2016.

[57] T. D. Na-Ayudhaya, S. Sakdarat, A. Shuyprom et al., "Chemical constituents of the leaves of Clinacanthus nutans Lindau," Journal of Phytopharmacy, vol. 8, no. 1, pp. 1-8, 2001.

[58] S. Sakdarat, A. Shuyprom, N. A. T. Dechatiwongse, P. G. Waterman, and G. Karagianis, "Chemical composition investigation of the Clinacanthus nutans Lindau leaves," Thai Journal of Phytopharmacy, vol. 13, no. 2, pp. 13-24, 2006.

[59] D. Huang, Y. Li, F. Cui, J. Chen, and J. Sun, "Purification and characterization of a novel polysaccharide-peptide complex from Clinacanthus nutans Lindau leaves," Carbohydrate Polymers, vol. 137, pp. 701-708, 2016.

[60] P. Kosai, K. Sirisidthi, and W. Jiraungkoorskul, "Evaluation of total phenolic compound and cytotoxic activity of Clinacanthus nutans," Indian Journal of Pharmaceutical Sciences, vol. 78, no. 2, pp. 283-286, 2016.

[61] S. Y. Lee, A. Mediani, A. H. Nur Ashikin, A. B. S. Azliana, and F. Abas, "Antioxidant and $\alpha$-glucosidase inhibitory activities of the leaf and stem of selected traditional medicinal plants," International Food Research Journal, vol. 21, no. 1, pp. 165-172, 2014.

[62] C. W. Mai, K. S. I. Yap, M. T. Kho et al., "Mechanisms underlying the anti-inflammatory effects of Clinacanthus nutans lindau extracts: Inhibition of cytokine production and toll-like receptor-4 activation," Frontiers in Pharmacology, vol. 7, no. 7, pp. 1-11, 2016.

[63] V. Thongrakard and T. Tencomnao, "Modulatory effects of Thai medicinal plant extract on proinflammatory cytokines-induced apoptosis in human keratinocyte HaCat cells," African Journal of Biotechnology, vol. 9, no. 31, pp. 4999-5003, 2010.

[64] J. M. P. Yuann, J. S. Wang, H. L. Jian, C. C. Lin, and J. Y. Liang, "Effects of Clinacanthus nutans (Burm. f) lindau leaf extracts on protection of plasmid DNA from riboflavin photoreaction," MC-Transaction on Biotechnology, vol. 4, no. 1, pp. 45-58, 2012 (Chinese).

[65] N. Uawonggul, A. Chaveerach, S. Thammasirirak, T. Arkaravichien, C. Chuachan, and S. Daduang, "Screening of plants acting against Heterometrus laoticus scorpion venom activity on fibroblast cell lysis," Journal of Ethnopharmacology, vol. 103, no. 2, pp. 201-207, 2006.
[66] N. Uawonggul, S. Thammasirirak, A. Chaveerach, C. Chuachan, J. Daduang, and S. Daduang, "Plant extract activities against the fibroblast cell lysis by honey bee venom," Journal of Medicinal Plants Research, vol. 5, no. 10, pp. 1978-1986, 2011.

[67] C. Thongharb and P. Tejasen, "The effect of Slaed Pang Porn (Clinacanthus nutans) on Thailand cobra venom (Naja naja siamensis)," Thai Journal of Pharmaceutical Sciences, vol. 2, pp. 1057-1063, 1977.

[68] S. Kittisiripornkul and K. Witthayāsāt, The Antiinflammatory Action and Toxicological Studies of Extracts from Clinacanthus nutans, Mahidol University, Bangkok, 1984.

[69] J. Satatyavivad, N. Bunyaprephatsara, S. Kitisiripornkul, and W. Tanasomwang, "Analgesic and anti-inflammatory activities of extract of Clinacanthus nutans (Burm.F.) Lindau," Thai Journal of Pharmceutical Sciences, vol. 3, no. 1, pp. 7-17, 1996.

[70] P. Wanikiat, A. Panthong, P. Sujayanon, C. Yoosook, A. G. Rossi, and V. Reutrakul, "The anti-inflammatory effects and the inhibition of neutrophil responsiveness by Barleria lupulina and Clinacanthus nutans extracts," Journal of Ethnopharmacology, vol. 116, no. 2, pp. 234-244, 2008.

[71] S. Suntararuks, The Study of Immunotoxic Effect of Clinacanthus nutans Extract in Male Wistar Rats, Mahidol University, 1999.

[72] W. Charerntantanakul and R. Kawaree, "Effects of medicinal plants extracts on interleukin-10 and tumor necrosis factor alpha gene expressions in porcine peripheral blood mononuclear cells," Chiang Mai Veterinary Journal, vol. 8, no. 2, pp. 93103, 2010.

[73] B. Sriwanthana, P. Chavalittumrong, and L. Chompuk, "Effect of Clinacanthus nutans on human cell-mediated immune response in vitro," Thailand Journal of Pharmaceutical Science, vol. 20, no. 4, pp. 261-267, 1996.

[74] C. S.-H. Tan, C. F.-Y. Ho, S.-S. Heng et al., "Clinacanthus nutans extracts modulate epigenetic link to cytosolic phospholipase A2 expression in SH-SY5Y cells and primary cortical neurons," NeuroMolecular Medicine, vol. 18, no. 3, pp. 441-452, 2016.

[75] H.-D. Tsai, J.-S. Wu, M.-H. Kao et al., "Clinacanthus nutans protects cortical neurons against hypoxia-induced toxicity by downregulating HDAC1/6," NeuroMolecular Medicine, vol. 18, no. 3, pp. 274-282, 2016.

[76] J.-S. Wu, M.-H. Kao, H.-D. Tsai et al., “Clinacanthus nutans mitigates neuronal apoptosis and ischemic brain damage through augmenting the C/EBP $\beta$-driven PPAR- $\gamma$ transcription," Molecular Neurobiology, pp. 1-14, 2017.

[77] K. W. Lau, S. K. Lee, and J. H. Chin, "Effect of the methanol leaves extract of Clinacanthus nutans on the activity of acetylcholinesterase in male mice," Journal of Acute Disease, vol. 3, no. 1, pp. 22-25, 2014.

[78] M. T. Chomnawang, S. Surassmo, V. S. Nukoolkarn, and W. Gritsanapan, "Effect of Garcinia mangostana on inflammation caused by Propionibacterium acnes," Fitoterapia, vol. 78, no. 6, pp. 401-408, 2007.

[79] S. Thongchai, T. Ekalaksananan, C. Pientong et al., "Anti-herpes simplex virus type 1 activity of crude ethyl acetate extract of Clinacanthus nutans," Journal of Science and Technology Mahasarakham University, vol. 27, no. 4, pp. 318-326, 2008.

[80] D. Thawaranantha, K. Balachandra, and S. Jongtrakulsiri, "In vitro antiviral activity of Clinacanthus nutans on varicella-zoster virus," Siriraj Hospital Gazette, vol. 44, no. 4, pp. 285-291, 1992.

[81] S. Charuwichitratana, N. Wongrattanapasson, P. Timpatanapong, and M. Bunjob, "Herpes zoster: Treatment with Clinacanthus nutans cream," International Journal of Dermatology, vol. 35, no. 9, pp. 665-666, 1996. 
[82] V. Thamlikitkul, "Treatment of herpes genitalis and herpes zoster with Clinacanthus nutans," Journal of Infectious Diseases and Antimicrobial Agents, pp. 95-96, 1995.

[83] P. Kunsorn, N. Ruangrungsi, V. Lipipun, A. Khanboon, and K. Rungsihirunrat, "The identities and anti-herpes simplex virus activity of Clinacanthus nutans and Clinacanthus siamensis," Asian Pacific Journal of Tropical Biomedicine, vol. 3, no. 4, pp. 284-290, 2013.

[84] C. Jayavasu, K. Balachandra, and S. Sangkitporn, "Clinical trial in the treatment of genital herpes patients with Clinacanthus nutans extract," Communicable Disease Journal, vol. 18, no. 3, pp. 152-161, 1992.

[85] S. Sangkitporn, B. Kroavon, P. Thawatsupha, M. Bunjob, and P. Chavalittumrong, "Treatment of recurrent genital herpes simplex virus infection with Clinacanthus nutans extract," Bulletin of the Department of Medical Service, vol. 18, pp. 226231, 1993.

[86] K. Chonlasin, S. Visetson, K. Phanwichien, and S. Foongladda, Ability of Clinacanthus nutans (Burm.F.) Lindau of inhibition Newcastle disease virus (NDV) infection in cell culture, Kasetsart University, 2002.

[87] S. Direkbusarakom, L. Ruangpan, Y. Ezura, and M. Yoshimizu, "Protective efficacy of Clinacanthus nutans on yellow-head disease in black tiger shrimp (Penaeus monodon)," Fish Pathology, vol. 33, no. 4, pp. 401-404, 1998.

[88] S. Arullappan, P. Rajamanickam, N. Thevar, and C. C. Kodimani, "In vitro screening of cytotoxic, antimicrobial and antioxidant activities of Clinacanthus nutans (Acanthaceae) leaf extracts," Tropical Journal of Pharmaceutical Research, vol. 13, no. 9, pp. 1455-1461, 2014.

[89] N. Cheeptham and G. H. N. Towers, "Light-mediated activities of some Thai medicinal plant teas," Fitoterapia, vol. 73, no. 7-8, pp. 651-662, 2002.

[90] S. Rathnasamy, K. B. Mohamed, S. F. Sulaiman, and A. Akinboro, "Evaluation of cytotoxic, mutagenic and antimutagenic potential of leaf extracts of three medicinal plants using Allium cepa chromosome assay," International Current Pharmaceutical Journal, vol. 2, no. 8, pp. 131-140, 2013.

[91] S. Timpawat and L. Vajrabhaya, "Clinical evaluation of Clinacanthus nutans Lindau in orabase in the treatment of recurrent aphthous stomatitis," Mahidol Dental Journal, vol. 14, no. 1, pp. 10-16, 1994.

[92] N. Pongphasuk, W. Khunkitti, and M. Chitcharoenthum, "Antiinflammatory and analgesic activities of the extract from Garcinia mangostana linn," Acta Horticulturae, vol. 680, pp. 125-130, 2005.

[93] W. L. V. Choy, K. K. Hwi, and N. Dublin, "65 Effects of Clinacanthus nutans and mebeverine hydrochloride on bladder activity," Journal of Men's Health, vol. 8, no. 1, pp. S119-S120, 2011.

[94] P. Pannangpetch, P. Laupattarakasem, V. Kukongviriyapan, U. Kukongviriyapan, B. Kongyingyoes, and C. Aromdee, "Antioxidant activity and protective effect against oxidative hemolysis of Clinacanthus nutans (Burm.f) Lindau," Songklanakarin Journal of Science and Technology, vol. 29, no. 1, pp. 1-9, 2007.

[95] S. Agustina, Isolation of Flavonoids as Antioxidant from Dandang Gendis (Clinacanthus nutans) Leaves, Bogor Agricultural University, 2011.

[96] C. Yoosook, Y. Panpisutchai, S. Chaichana, T. Santisuk, and V. Reutrakul, "Evaluation of anti-HSV-2 activities of Barleria lupulina and Clinacanthus nutans," Journal of Ethnopharmacology, vol. 67, no. 2, pp. 179-187, 1999.
[97] C. Jayavasu, T. Dechatiwongse, K. Balachandra, P. Chavalittumrong, and S. Jongtrakulsiri, "The virucidal activity of Clinacanthus nutans Lindau extracts against herpes simplex virus type-2: An in vitro study," Bulletin of the Department of Medical Sciences, vol. 34, no. 4, pp. 153-158, 1992.

[98] S. Direkbusarakom, A. Herunsalee, M. Yoshimizu, and Y. Ezura, "Antiviral activity of several Thai traditional herb extracts against fish pathogenic viruses," Fish Pathology, vol. 31, no. 4, pp. 209-213, 1996.

[99] S. Direkbusarakom, Y. Ezura, M. Yoshimizu, and A. Herunsalee, "Efficacy of Thai traditional herb extracts against fish and shrimp pathogenic bacteria," Fish Pathology, vol. 33, no. 4, pp. 437-441, 1998.

[100] M. T. Chomnawang, S. Surassmo, K. Wongsariya, and N. Bunyapraphatsara, "Antibacterial activity of Thai medicinal plants against methicillin-resistant Staphylococcus aureus," Fitoterapia, vol. 80, no. 2, pp. 102-104, 2009.

[101] M. T. Chomnawang, C. Trinapakul, and W. Gritsanapan, "In vitro antigonococcal activity of Coscinium fenestratum stem extract," Journal of Ethnopharmacology, vol. 122, no. 3, pp. 445449, 2009.

[102] M. T. Chomnawang, S. Surassmo, V. S. Nukoolkarn, and W. Gritsanapan, "Antimicrobial effects of Thai medicinal plants against acne-inducing bacteria," Journal of Ethnopharmacology, vol. 101, no. 1-3, pp. 330-333, 2005.

[103] E. Farsi, K. Esmailli, A. Shafaei et al., "Mutagenicity and preclinical safety assessment of the aqueous extract of Clinacanthus nutans leaves," Drug and Chemical Toxicology, vol. 545, pp. 1-13, 2016.

[104] K. Esmailli, F. Alsuede, A. Aisha, A. Shafaei, and Z. Ismail, "Preliminary phytochemical analysis and cytotoxicity studies of Clinacanthus nutans (Sabah snake grass)," The Open Conference Proceedings Journal, vol. 4, no. 3, 187 pages, 2013.

[105] S.-Y. Liew, E. J. Stanbridge, K. Yusoff, and N. Shafee, "Hypoxia affects cellular responses to plant extracts," Journal of Ethnopharmacology, vol. 144, no. 2, pp. 453-456, 2012.

[106] S. Y. Fong, T. Piva, C. Dekiwadia, S. Urban, and T. Huynh, "Comparison of cytotoxicity between extracts of Clinacanthus nutans (Burm. f.) Lindau leaves from different locations and the induction of apoptosis by the crude methanol leaf extract in D24 human melanoma cells," BMC Complementary and Alternative Medicine, vol. 16, no. 368, pp. 1-12, 2016.

[107] S. Sakdarat, A. Shuyprom, C. Pientong, T. Ekalaksananan, and S. Thongchai, "Bioactive constituents from the leaves of Clinacanthus nutans Lindau," Bioorganic \& Medicinal Chemistry, vol. 17, no. 5, pp. 1857-1860, 2009.

[108] S. Sittiso, T. Ekalaksananan, and C. Pientong, "Effects of compounds from Clinacanthus nutans on dengue virus type 2 infection," Srinagarind Medical Journal, vol. 25, pp. 272-275, 2010. 


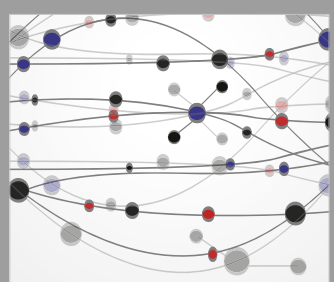

The Scientific World Journal
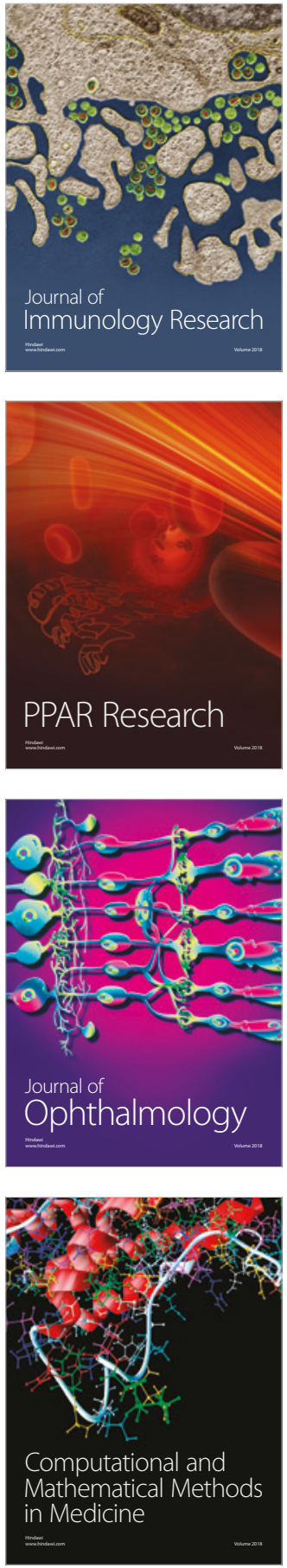

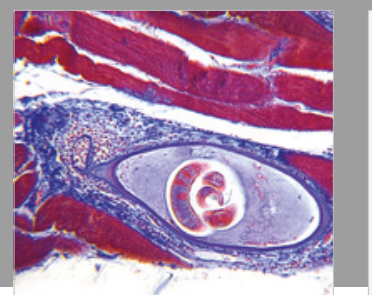

Gastroenterology Research and Practice

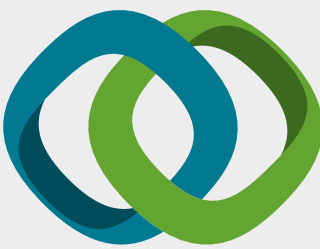

\section{Hindawi}

Submit your manuscripts at

www.hindawi.com
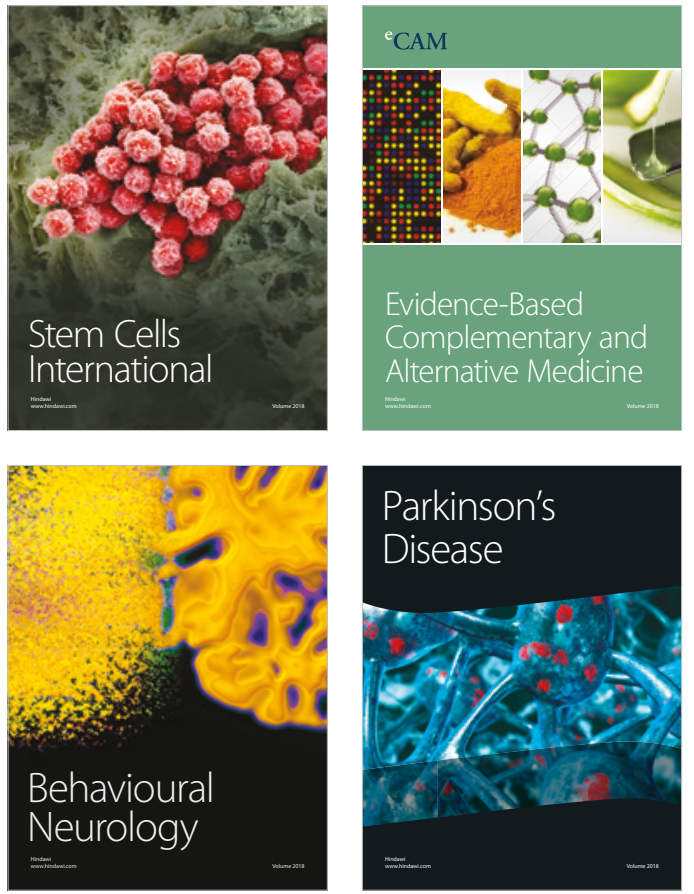

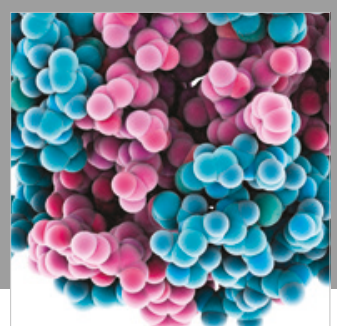

ournal of

Diabetes Research

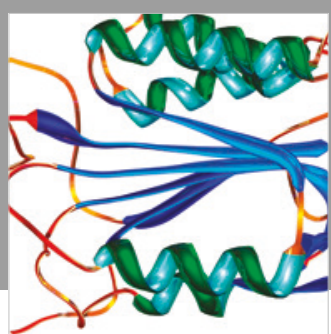

Disease Markers
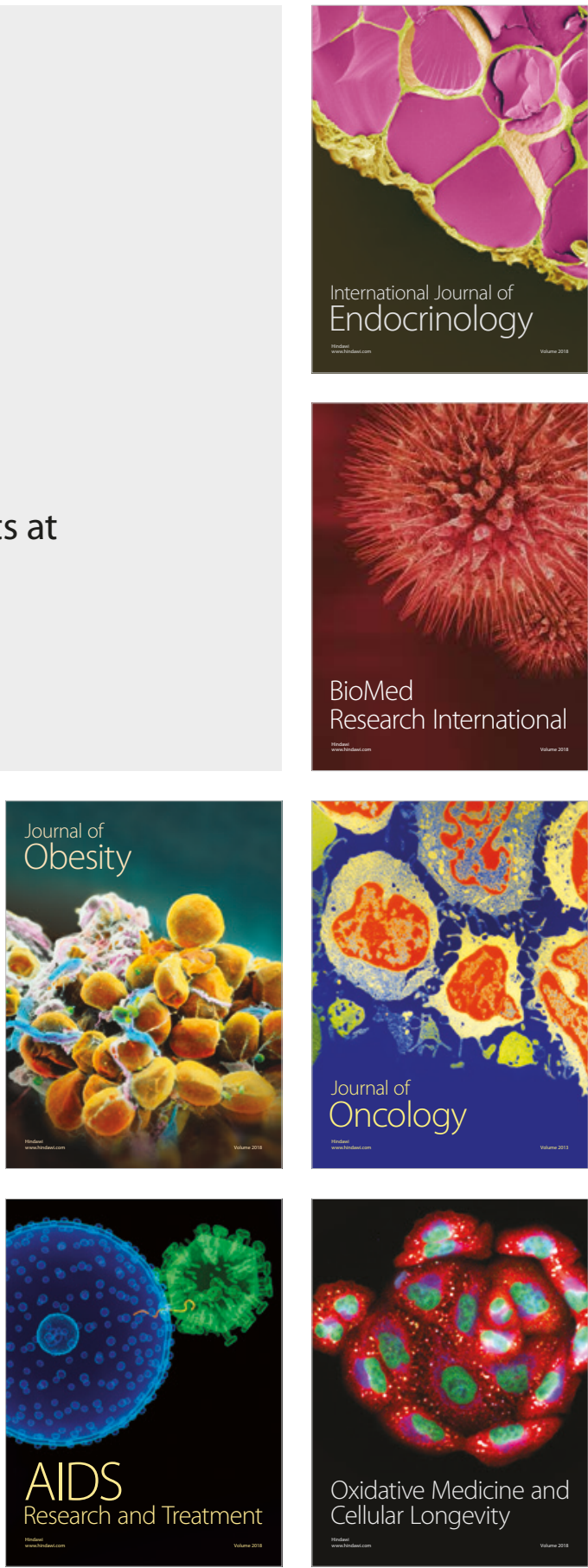